\title{
Genome-wide identification and analysis of DNA methyltransferase and demethylase gene families in Dendrobium officinale reveal their potential functions in polysaccharide accumulation
}

Zhenming $\mathrm{Yu}^{1,2}$, Guihua Zhang ${ }^{1}$, Jaime A. Teixeira da Silva ${ }^{3}$, Mingzhi $\mathrm{Li}^{4}$, Conghui Zhao ${ }^{1}$, Chunmei $\mathrm{He}^{1}$, Can $\mathrm{Si}^{1}$, Mingze Zhang ${ }^{1}$ and Jun Duan ${ }^{1,2^{*}}$

\begin{abstract}
Background: DNA methylation is a conserved and important epigenetic modification involved in the regulation of numerous biological processes, including plant development, secondary metabolism, and response to stresses. However, no information is available regarding the identification of cytosine-5 DNA methyltransferase (C5-MTase) and DNA demethylase (dMTase) genes in the orchid Dendrobium officinale.

Results: In this study, we performed a genome-wide analysis of DoC5-MTase and DodMTase gene families in D. officinale. Integrated analysis of conserved motifs, gene structures and phylogenetic analysis showed that eight DoC5-MTases were divided into four subfamilies (DoCMT, DoDNMT, DoDRM, DoMET) while three DodMTases were divided into two subfamilies (DoDML3, DoROS1). Multiple cis-acting elements, especially stress-responsive and hormone-responsive ones, were found in the promoter region of DoC5-MTase and DodMTase genes. Furthermore, we investigated the expression profiles of DoC5-MTase and DodMTase in 10 different tissues, as well as their transcript abundance under abiotic stresses (cold and drought) and at the seedling stage, in protocorm-like bodies, shoots, and plantlets. Interestingly, most DoC5-MTases were downregulated whereas DodMTases were upregulated by cold stress. At the seedling stage, DoC5-MTase expression decreased as growth proceeded, but DodMTase expression increased.
\end{abstract}

Conclusions: These results provide a basis for elucidating the role of DoC5-MTase and DodMTase in secondary metabolite production and responses to abiotic stresses in D. officinale.

Keywords: Dendrobium officinale, DNA methyltransferase, Stress, Water-soluble polysaccharides

\footnotetext{
* Correspondence: duanj@scib.ac.cn

${ }^{1}$ Key Laboratory of South China Agricultural Plant Molecular Analysis and Genetic Improvement, South China Botanical Garden, Chinese Academy of Sciences, Guangzhou 510650, China

${ }^{2}$ Economic Botany, Core Botanical Gardens, Chinese Academy of Sciences, Guangzhou 510650, China

Full list of author information is available at the end of the article
}

(c) The Author(s). 2021 Open Access This article is licensed under a Creative Commons Attribution 4.0 International License, which permits use, sharing, adaptation, distribution and reproduction in any medium or format, as long as you give appropriate credit to the original author(s) and the source, provide a link to the Creative Commons licence, and indicate if changes were made. The images or other third party material in this article are included in the article's Creative Commons licence, unless indicated otherwise in a credit line to the material. If material is not included in the article's Creative Commons licence and your intended use is not permitted by statutory regulation or exceeds the permitted use, you will need to obtain permission directly from the copyright holder. To view a copy of this licence, visit http://creativecommons.org/licenses/by/4.0/ The Creative Commons Public Domain Dedication waiver (http://creativecommons.org/publicdomain/zero/1.0/) applies to the data made available in this article, unless otherwise stated in a credit line to the data. 


\section{Background}

Cytosine DNA methylation is an evolutionarily conserved epigenetic modification that is critical for the regulation of gene expression, imprinting, plant development, fruit ripening, control of transposon activity, and stress responses [1-3]. Mammalian methylation mainly occurs in symmetric CG contexts, while plant DNA methylation occurs in all sequence contexts, including CG, CHG, and $\mathrm{CHH}$, where $\mathrm{H}$ represents $\mathrm{A}, \mathrm{C}$, or $\mathrm{T}$ [4]. In plants, DNA methylation is divided into two categories: maintenance of DNA methylation and de novo DNA methylation. Plant cytosine DNA methylation can be maintained by METHYLTRANSFERASE1 (MET1), CHROMOMETHYLASE3 (CMT3), and DOMAINS REARRANGED METHYLASE2 (DRM2) and CMT2 at $\mathrm{CG}, \mathrm{CHG}$, and $\mathrm{CHH}$ sites, respectively [2-6]. In addition, DRM is primarily responsible for de novo DNA methylation in all sequence contexts (CG, CHG, and $\mathrm{CHH}$ ) via the DNA methylation pathway directed by 24-nt small interfering RNAs [3, 4].

Generally, the precise status of DNA methylation in plants depends on the dynamics of antagonistic DNA methylation and demethylation. Compared to DNA methylation, whose catalyzation is determined by a single DNA methyltransferase such as MET1, CMT2, CMT3 or DRM2, active DNA demethylation requires a series of enzymes involved in excision of 5methylcytosine in the CG, CHG and $\mathrm{CHH}$ contexts [2]. In plants, this process is initially catalyzed by a bifunctional DNA glycosylase, including REPRESSOR OF SILE NCING1 (ROS1), DEMETER (DME), DEMETER-like 2 (DML2), or DEMETER-like 3 (DML3), through the base-excision repair pathway $[1,3]$.

Increasing evidence demonstrates that plant DNA methylation and demethylation are closely associated with various environmental stresses, including cold [7], drought [8], heat [9], heavy metal [10], salt [11], and ultraviolet stresses [12]. Additionally, DNA methylation and demethylation also play an indispensable role in regulating fruit development and ripening [3, 13, 14], as well as secondary metabolism [15-17]. Differences in anthocyanin levels between Malus domestica 'Granny Smith' and 'Golden Delicious' are attributed to differential DNA methylation levels of the v-myb avian myeloblastosis viral oncogene homolog (MdMYB1) promoter in both cultivars, altering the differential accumulation of MdMYB1-specific transcript levels, and in turn affecting the formation of red pigmentation in apple skin [18]. Similarly, temperature-dependent DNA demethylation is a key factor of postharvest temperature-affected anthocyanin biosynthesis in the flesh of an originally whitefleshed peach (Prunus persica). Increased expression levels of the anthocyanin biosynthesis-related genes, including phenylalanine ammonia-lyase (PpPAL), cinnamate 4-hydroxylase $(\mathrm{PpC} 4 \mathrm{H})$, 4-coumarate coenzyme $A$ ligase (Pp4CL), flavanone 3-hydroxylase $(\mathrm{PpF} 3 \mathrm{H})$, flavonoid 3'-hydroxylase $(\mathrm{PpF3} H)$, dihydroflavonol 4-reductase (PpDFR) and anthocyanin synthase $(P p A N S)$, as well as a transcription factor gene, basic helix-loop-helix 3 (PpbHLH3), were associated with lower methylation levels in the promoters of these genes [19]. Additionally, OsROS1-mediated DNA demethylation in the rice endosperm restricts the number of aleurone cell layers, and thus the OsROS1 mutant contains more non-starch polysaccharides, lipids, proteins, vitamins, and minerals than the wild type [20]. Given that DNA methylation and demethylation are essential for many biological processes in plants, C5-MTase and dMTase genes have already been identified and characterized in several plant species, such as thale cress (Arabidopsis thaliana) [21], peanut (Arachis hypogaea) [22], rapeseed (Brassica napus) [23], castor bean (Ricinus communis) [24], tomato (Solanum lycopersicum) [25], and tea plant (Camellia sinensis) [26]. However, to date, no research has yet focused on the identification and analysis of C5-MTase and dMTase genes in the orchid, Dendrobium officinale Kimura et Migo, based on genome-wide analyses.

D. officinale belongs to the Orchidaceae, and is a timehonored tonic food and traditional Chinese herbal medicine because of its abundant active secondary metabolites found in stems, especially water-soluble polysaccharides (WSPs) that exhibit anti-inflammatory, antitumor and antioxidant activities [27, 28]. Previous studies indicated that $D$. officinale WSPs can improve abiotic stress (e.g. drought and salt stress) tolerance, because they can act as compatible solutes and enhance water uptake from an osmotically stressed environment [29-31]. In addition, most epiphytic orchids adhere tightly to the surface of tree bark or rocks, so that they usually experience environmental stresses (e.g. cold and heat stress, and water deficit). Hence, D. officinale has evolved desirable qualities for mitigating harsh habitats, for instance Crassulacean acid metabolism, pseudobulbs, succulent storage organs, and thick leaves [32]. The $D$. officinale reference genome is publicly available [33, 34]. Several bioactive compounds with medicinal effects, such as polysaccharides and alkaloids, have been identified and functionally analyzed [28-30, 35, 36]. However, no information on C5-MTase and dMTase genes in D. officinale is available. In order to investigate whether DNA methylation and demethylation are involved in the regulation of the biosynthesis of active compounds, genome-guided discovery and characterization of C5MTase and dMTase genes in D. officinale were performed. Our study provides valuable information for future functional characterization of these two epigenetic regulatory enzymes in plants of the orchid $D$. officinale, 
as well as for analyzing their evolutionary relationships within the entire plant kingdom.

\section{Results}

Genome-wide identification and structural analysis of $C 5$ MTase and dMTase genes in D. officinale

Blast analysis of reported A. thaliana and rice C5MTase and dMTase proteins against the whole D. officinale genome resulted in the identification of eight DoC5-MTase and three DodMTase proteins (Table 1). The eight DoC5-MTase genes (DoMET1, DoCMT1, DoCMT2, DoCMT3, DoDRM1, DoDRM2, DoDRM3 and DoDNMT2) code proteins composed of 324 (DoDNMT2) to 1534 (DoMET1) amino acids, similar to AtC5-MTases (383 to 1534) [21]. The MW of DoC5MTases varies from 36.43 to $173.71 \mathrm{kDa}$ with a $\mathrm{pI}$ ranging from 5.00 to 9.16 (Table 1). The full-length of the three DodMTase proteins varies from 1380 (DoDML3) to 1903 (DoROS1b) amino acids with an open reading frame (ORF) ranging from 4143 to $5712 \mathrm{bp}$, relative MW between 156.56 and $212.56 \mathrm{kDa}$, and a pI ranging from 6.70 to 7.85 (Table 1). The highest GRAVY value was observed for DoDNMT2 $(-0.235)$ and the lowest value for DoCMT1 (-0.626), indicating that all $D$. officinale DoC5-MTase and DodMTase proteins are hydrophilic (Table 1). According to their putative subcellular localization, DoDRM1 was located in the cytoplasm, whereas the other 10 proteins were located in the nucleus. These findings are consistent with results in Ricinus communis in which different DRM members are located in different organelles [24]. Apart from the nuclear localization signals, RcDRM3 may be located in the mitochondrion, and RcDRM1 may be located in the chloroplast or mitochondrion [24].
The sequence similarity of $D$. officinale DoC5-MTase and DodMTase proteins was also analyzed. Compared with DodMTase proteins, DoC5-MTase proteins displayed low identity with each other, indicating that different DoC5-MTase proteins might possess diverse functions. DoCMT1 and DoCMT3, as well as DoDRM1 and DoDRM2, shared significantly higher similarities than other DoC5-MTase proteins. Among DodMTase proteins, sequence similarity between DoROS1a and DoROS1b was highest (Fig. 1). These results suggest that some sequences may be duplicated in DoC5-MTase and DodMTase genes. In addition, the coding region of DoC5-MTase genes was interrupted by $5-28$ introns, while that of DodMTase was interrupted by 34-39 introns. Among these, the DodMTase gene with the largest number of introns is DoDML3, while DoDRM1 contains the least introns (Fig. 2).

Although the catalytic C-terminal domains with conserved domains were ubiquitous in all identified DoC5MTase and DodMTase proteins, the N-terminals showed diverse combinations of conserved domains in each subfamily. Structural analysis revealed that eight DoC5-MTase proteins were divided into four groups on the basis of the diverse structural features comprising their primary structure: DoMET1 comprised the MET group that harbored two $\mathrm{BAH}$ domains in the $\mathrm{N}$ terminal, DoCMT1, DoCMT2 and DoCMT3 comprised the CMT group that harbored only one BAH in the Nterminal, DoDRM1, DoDRM2 and DoDRM3 comprised the DRM group that harbored the UBA domain in the $\mathrm{N}$-terminal, while DoDNMT2 was the sole member in the DNMT group without an N-terminal conserved domain. All of the DoC5-MTase proteins contained the carboxyl C-terminal catalytic domain with the conserved motifs I, IX, VI, VIII, IV and X aligned in a specific

Table 1 Basic features of C5-MTase and dMTase genes identified in D. officinale

\begin{tabular}{|c|c|c|c|c|c|c|c|c|c|}
\hline $\begin{array}{l}\text { Gene } \\
\text { name }\end{array}$ & Gene ID & $\begin{array}{l}\text { Chromosome } \\
\text { location }\end{array}$ & $\begin{array}{l}\mathrm{ORF}^{\mathrm{a}} \\
(\mathrm{bp})\end{array}$ & $\begin{array}{l}A A^{b} \\
\text { (aa) }\end{array}$ & $\begin{array}{l}M^{c} \\
(k D a)\end{array}$ & $\begin{array}{l}\text { GRAVYd } \\
\text { value }\end{array}$ & $\mathrm{pl}^{\mathrm{e}}$ & Intron $^{f}$ & $\begin{array}{l}\text { Predicted subcellular } \\
\text { localization }\end{array}$ \\
\hline DOMET1 & Dca001068 & Scaff_scaffold_716 & 4605 & 1534 & 173.71 & -0.414 & 5.76 & 18 & Nuclear \\
\hline DoCMT1 & Dca005086 & Scaff_scaffold_370 & 2883 & 960 & 107.69 & -0.626 & 6.24 & 26 & Nuclear \\
\hline DoCMT2 & Dca003680 & Scaff_scaffold_390 & 975 & 324 & 36.43 & -0.238 & 7.49 & 7 & Nuclear \\
\hline DoCMT3 & Dca021108 & Scaff_scaffold_883 & 2670 & 889 & 100.30 & -0.543 & 5.34 & 28 & Nuclear \\
\hline DoDRM1 & Dca019831 & Scaff_scaffold_547 & 1425 & 474 & 53.70 & -0.367 & 9.16 & 5 & Cytoplasmic \\
\hline DoDRM2 & Dca002179 & Scaff_scaffold_1220 & 1749 & 582 & 65.32 & -0.469 & 5.00 & 12 & Nuclear \\
\hline DoDRM3 & Dca020469 & Scaff_scaffold_726 & 1473 & 527 & 59.32 & -0.416 & 6.18 & 17 & Nuclear \\
\hline DoDNMT2 & Dca009405 & Scaff_scaffold_264 & 1053 & 350 & 40.11 & -0.235 & 6.60 & 13 & Nuclear \\
\hline DoDML3 & Dca014673 & Scaff_scaffold_807 & 4143 & 1380 & 156.56 & -0.597 & 7.85 & 39 & Nuclear \\
\hline DoROS1a & Dca024052 & Scaff_scaffold_489 & 5568 & 1855 & 207.52 & -0.592 & 7.01 & 37 & Nuclear \\
\hline DoROS1b & Dca020504 & Scaff_scaffold_993 & 5712 & 1903 & 212.56 & -0.603 & 6.70 & 34 & Nuclear \\
\hline
\end{tabular}

${ }^{\mathrm{a}} \mathrm{ORF}$, open reading frame; ${ }^{\mathrm{b}} \mathrm{AA}$, amino acid; ${ }^{\mathrm{C}} \mathrm{MW}$, molecular weight; ${ }^{\mathrm{d}} \mathrm{GRAVY}$, grand average of hydrophobicity; ${ }^{\mathrm{e}}$ pl, theoretical isoelectric point; ${ }^{\mathrm{f}}$ Intron, number of introns. Subcellular localization was predicted by Plant-mPLoc (http://www.csbio.sjtu.edu.cn/bioinf/plant-multi/) 


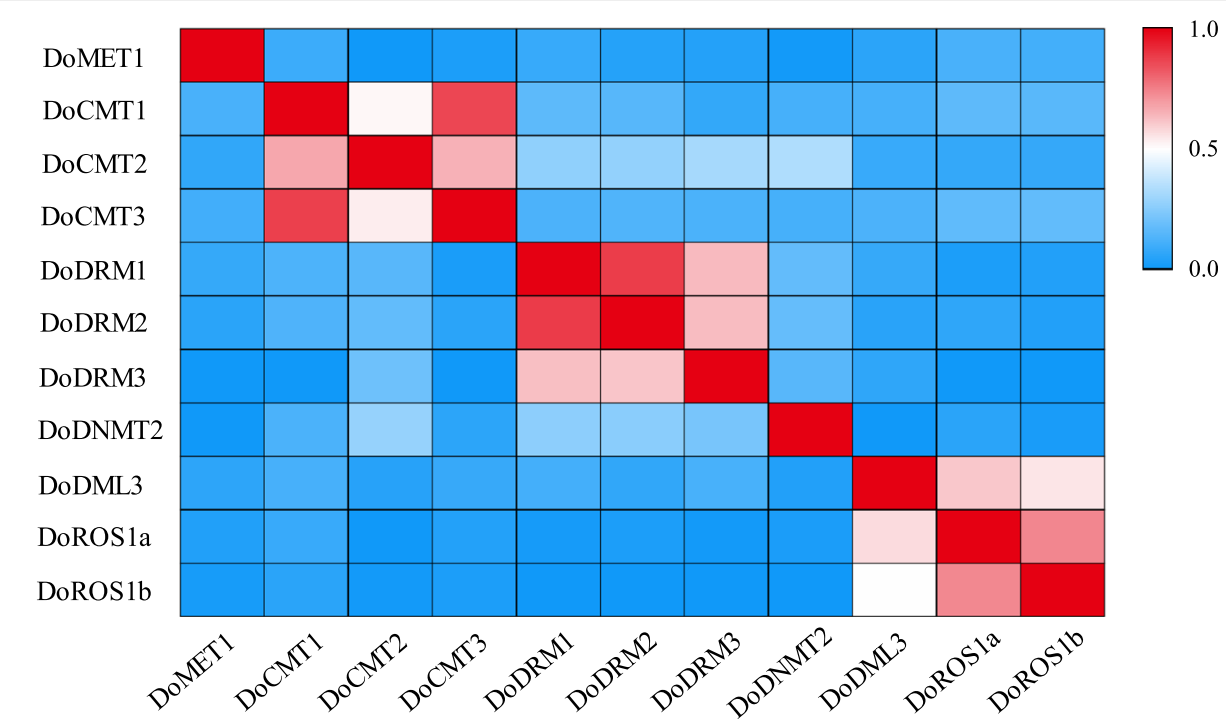

Fig. 1 Sequence similarity of DoC5-MTases and DodMTases in D. officinale. Color scale on the right indicates the correlation of two proteins. Dodger blue represents a weak correlation, while deep red represents a strong correlation for these proteins

order, whereas the divergence of the $\mathrm{N}$-terminal domains could be critical for distinct roles of different DoC5-MTase proteins (Fig. 3, Figure S1, S2, S3, S4, S5 and S6). In addition, DodMTase proteins contained three conserved domains, HhH-GPD, FES and RRMDME. These results suggest that the majority of domains in DodMTase proteins are highly conserved.

\section{Sequence alignment and conserved motif analysis of C5- MTases and dMTases in D. officinale}

To further explore the conservation and divergence of DoC5-MTase and DodMTase proteins, the MEME motif search tool was used to identify conserved motifs in $D$. officinale and $A$. thaliana, a dicotyledonous model plant, and O. sativa, a monocotyledonous model plant. Among these C5-MTase proteins, a total of 10 conserved motifs were identified. The length of motifs ranged from 29 to
50 amino acids, and the number of motifs varied between 1 (DoDNMT2) and 10 (AtCMT2). Motifs 6, 8 and 10 were highly conserved in the DRM subfamily. Motifs $1,2,3,4,5$ and 9 were located in the C-terminal region, and were the major conserved motifs in the CMT and MET subfamilies. Apart from OsCMT3, AtDRM3 and DoDRM3, motif 3 was located in all C5-MTase proteins (Fig. 4a, Figure S7, Table S2).

Accordingly, the distribution of dMTase protein motifs in A. thaliana, D. officinale and O. sativa were analyzed using the MEME suite, and 15 conserved motifs, designated as motifs 1 to 15, were identified. The length of motifs ranged from 18 to 50 amino acids and the number of motifs in each dMTase protein varied between 10 and 15 . Motifs $1,2,4$ and 12 were highly conserved in all dMTase proteins. Motifs 3, 6, 7, 8, 9 and 15 were found in 12 of $13 \mathrm{dMTase}$ proteins, motifs 5,11 and 14

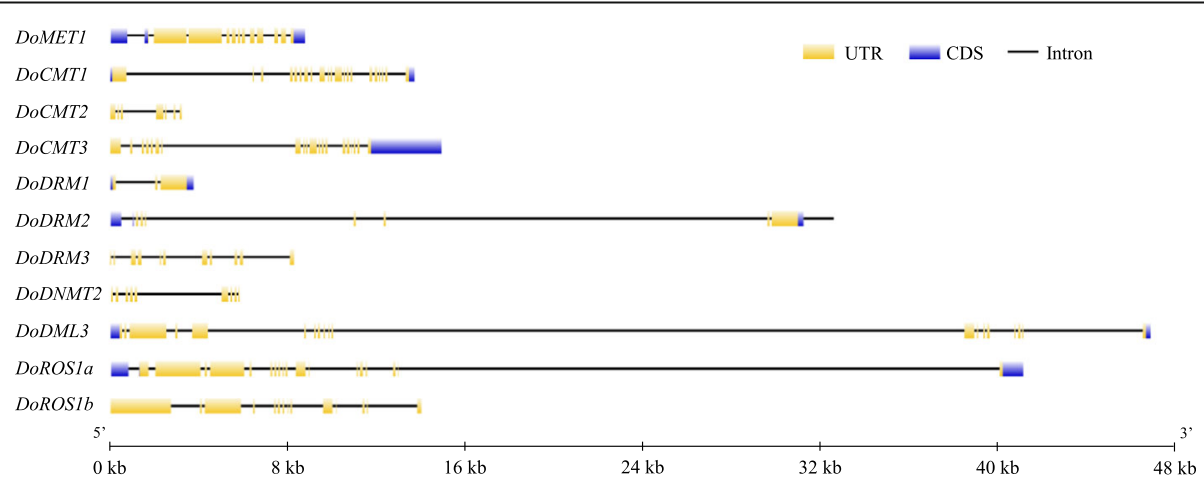

Fig. 2 Gene structures of DoC5-MTase and DodMTase genes in D. officinale. Introns are presented by lines. UTR and CDS are indicated by filled yellow and blue boxes, respectively 


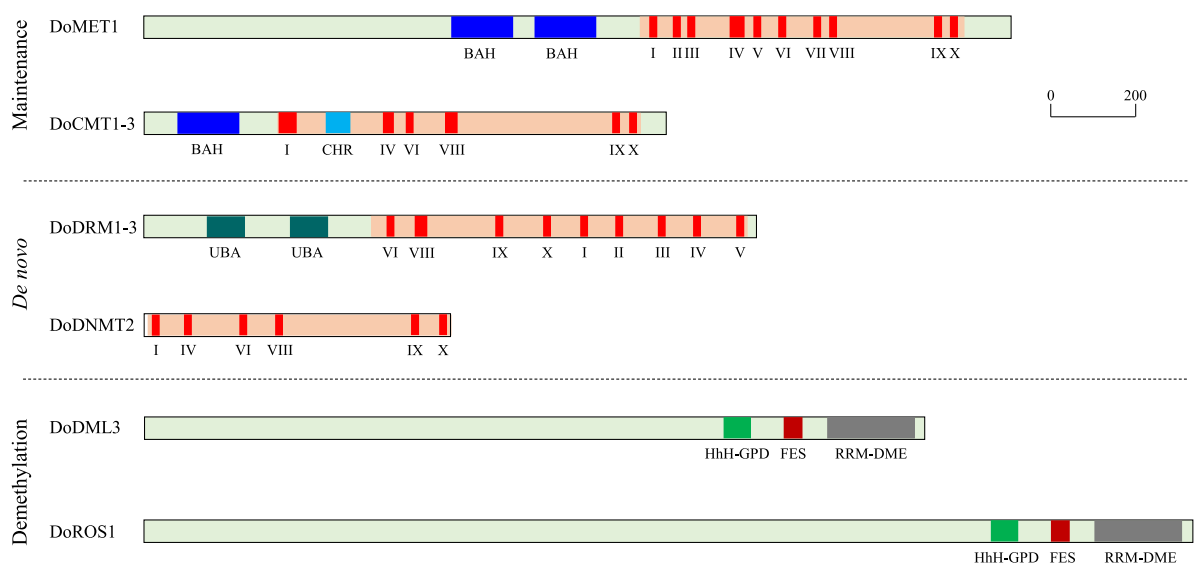

Fig. 3 Schematic structures of DoC5-MTase and DodMTase proteins in D. officinale. BAH, bromo adjacent homology domain; CHR, chromo domain; FES, 4Fe-4S cluster domain; HhH-GDP, helix-hairpin-helix-Gly-Pro-Asp domain; RRM-DME, RNA-recognition motif demethylase PF15628 in Demeter; UBA, ubiquitin-associated domain PF00627. I to X, the conserved motifs in C5-MTase

were observed in 11 of 13 dMTase proteins, and motifs 10 and 13 were observed in 10 of 13 dMTase proteins (Fig. 4b, Figure S8, Table S3). It could be inferred that motifs common to both C5-MTase and dMTase proteins are probably associated with conserved biological functions, but those specific to a few proteins may be related to gene-specific functions.

\section{Phylogenetic analysis of C5-MTases and dMTases in D. officinale and other plant species}

To elucidate the phylogenetic relationship among C5MTases in plants, 103 C5-MTase protein sequences from five monocotyledons ( $B$. distachyon, D. officinale, $O$. sativa, S. bicolor and $Z$. mays) and seven dicotyledons (A. lyrata, A. thaliana, E. guttata, P. trichocarpa, R. communis, S. lycopersicum and S. miltiorrhiza) were used to construct corresponding phylogenetic trees (Table S4). C5-MTases were naturally grouped into four subfamilies, including 35, 32, 12 and 24 members in CMT, DRM, MET and DNMT groups, respectively (Fig. 5a). Compared to the DRM subfamily, the MET, CMT and DNMT subfamilies were more similar and belonged to the same cluster. The DRM subfamily was the largest in the phylogenetic tree, whereas the DNMT subfamily was the smallest and only contained 12 members from the 12 plant species. The MET subfamily harbored DoMET1 and could be further divided into dicot and monocot groups. Similarly, CMT, DRM and DNMT2 subfamilies could also be divided into a monocot group and a dicot group.

Accordingly, 69 dMTase proteins were clustered into three orthologous clades, designated as DME, DML3 and ROS1 groups, which harbored 11, 22 and 36 members, respectively (Fig. 5b, Table S5). According to the constructed phylogenetic tree, the ROS1 and DML3 groups could be further divided into the monocot and dicot subgroups. However, the DME group was only restricted to dicots, indicating that DME might be phylogenetically monophyletic in dicots. Overall, these findings suggest that C5-MTase and dMTase proteins might exercise different functions in monocots and dicots.

Protein-protein interaction of DoC5-MTase and DodMTase Using A. thaliana homologues, a protein-protein interaction network of DoC5-MTase and DodMTase proteins was constructed with the STRING 11 tool. Consequently, DoC5-MTase and DodMTase were aligned to the corresponding $A$. thaliana orthologous proteins (Fig. 6). DoROS1a and DoROS1b were highly homologous to AtROS1, with 56 and 42\% identity, respectively. A strong interaction among CMTs, DRMs and METs was observed at a given confidence level (0.70), indicating that they might regulate the overall DNA methylation levels via a protein-protein interaction network or by forming protein complexes. ROS1s and DMLs interact with C5-MTases, especially CMTs and DRMs, indicating that global DNA methylation levels might be dynamically accommodated by both C5-MTase and dMTase. Consequently, C5-MTase and dMTase might form a reciprocal negative feedback loop, which dynamically affects the overall methylation levels.

\section{Analysis of cis-acting elements in DoC5-MTase and DodMTase genes}

Plant growth and development are regulated by different cis-regulatory elements in genes. Here, various cis-acting elements, containing hormone-sensitive, light-responsive, stress-responsive, tissue-specific and other elements were mined and analyzed from the 2000-bp upstream regulatory regions of DoC5-MTase and DodMTase genes using the PlantCARE web site (Fig. 7, Figure S9, Table S6). 

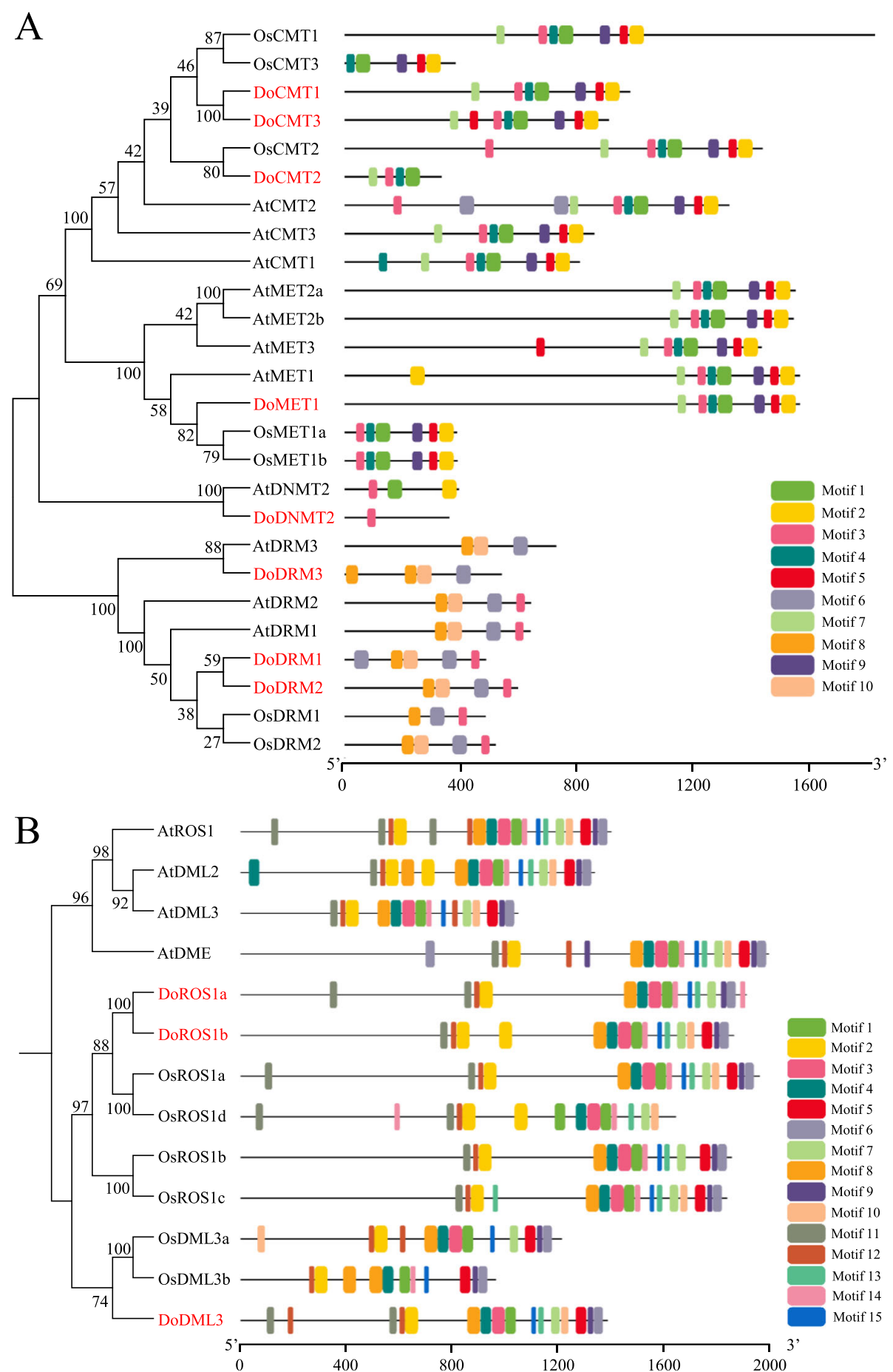

Fig. 4 Conserved motifs of C5-MTase (a) and dMTase (b) proteins from D. officinale, A. thaliana and O. sativa

Tissue-specific elements (12/244), including in the endosperm (6/12), shoot and root meristem (5/12) and seed (1/ $12)$, were found in the putative promoters of DoC5-MTase and DodMTase genes. Hormone-responsive elements (70/ $244)$, in response to ABA (18/70), auxin (9/70), ethylene (6/70), GA (18/70), MeJA (18/70) and SA (1/70), were also widely observed. Multiple abiotic and biotic stress-related elements $(69 / 244)$, including in response to anaerobic induction (11/69), dehydration (6/69), drought (1/69), heat $(2 / 69)$, low temperature (17/69), stress (15/69) and wounding (17/69), were largely enriched. These findings suggest that the DoC5-MTase and DodMTase genes might 


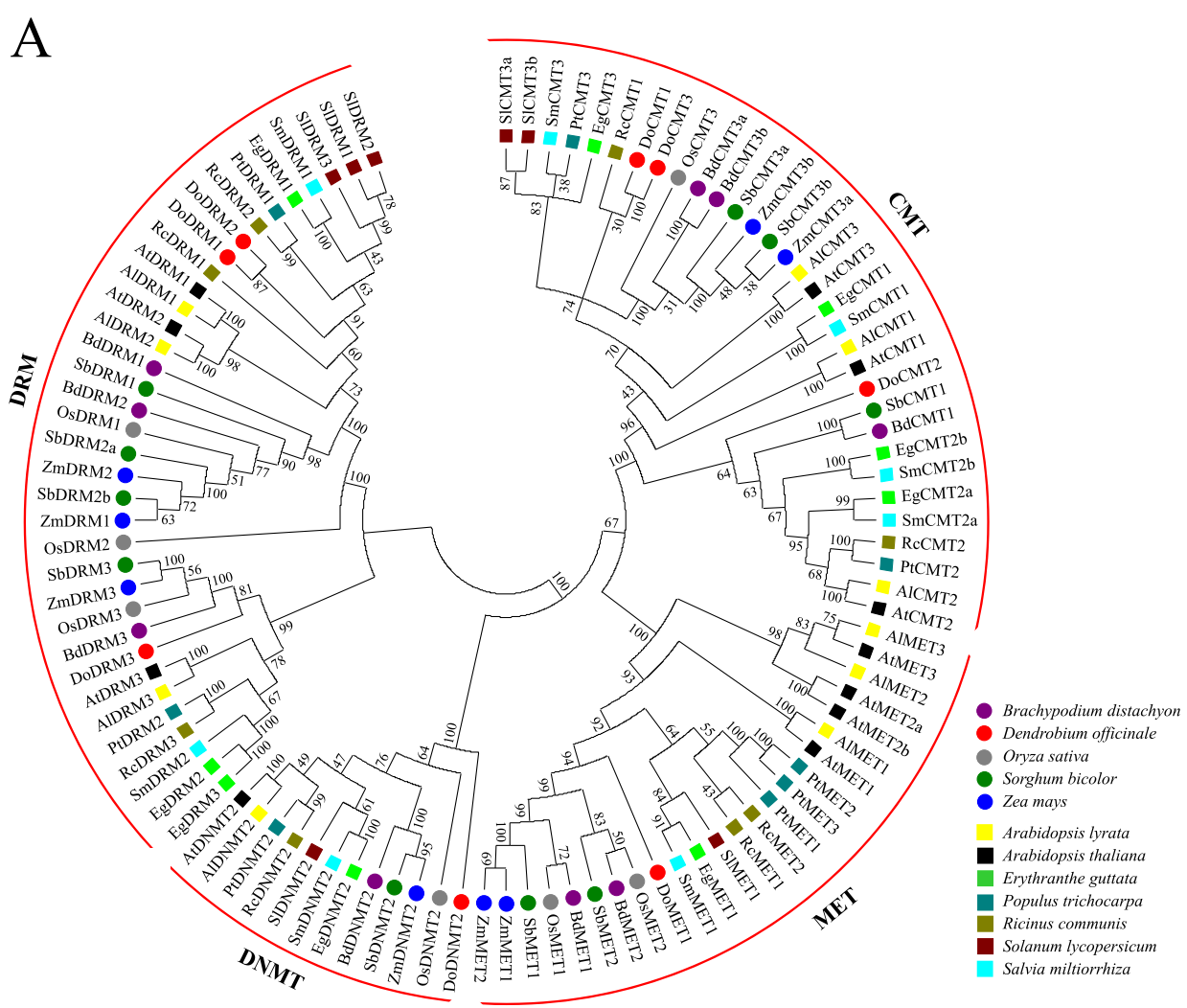

B

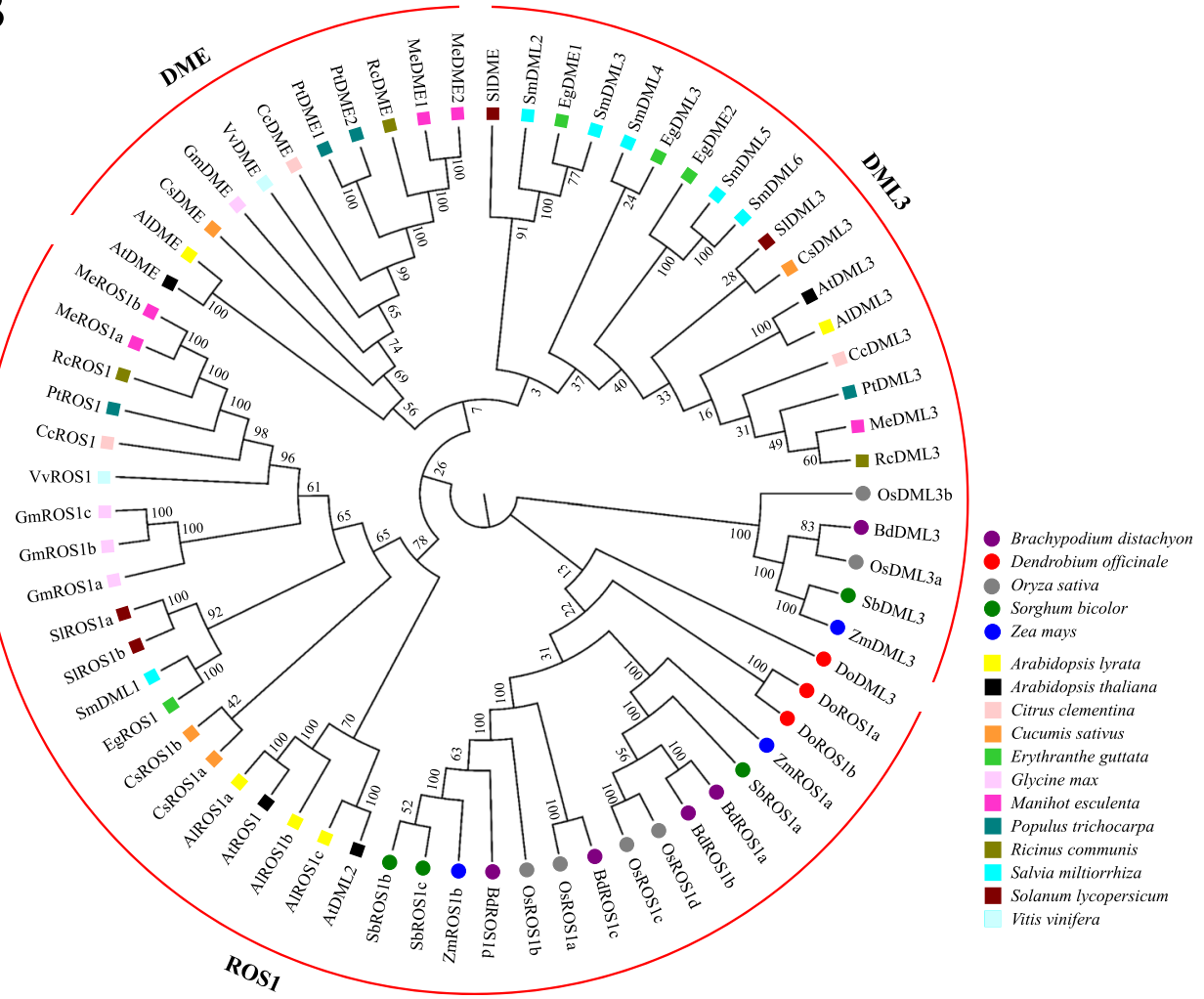

Fig. 5 (See legend on next page.) 
(See figure on previous page.)

Fig. 5 Phylogenetic analysis of the C5-MTase (a) and dMTase (b) proteins. Dicots and monocots are shown by a circle and square, respectively. Al, Arabidopsis lyrata; At, Arabidopsis thaliana; Bd, Brachypodium distachyon; Cc, Citrus clementina; Cs, Cucumis sativus; Do, Dendrobium officinale; Eg, Erythranthe guttata; Gm, Glycine max; Me, Manihot esculenta; Os, Oryza sativa; Pt, Populus trichocarpa; Rc, Ricinus communis; Sm, Salvia miltiorrhiza; $\mathrm{SI}$, Solanum lycopersicum; Sb, Sorghum bicolor; Vv, Vitis vinifera; Zm, Zea mays

play an important role in response to cold and drought stresses in D. officinale.

\section{Transcript abundance analysis of DoC5-MTase and} DodMTase genes in $D$. officinale

As the main participants of DNA methylation, C5MTases and dMTases play important roles in plant growth and development. To preliminarily investigate the biological function of DoC5-MTases and DodMTases, the transcript abundance of eight DoC5-MTase and three DodMTase genes in flower buds, green root tips, gynostemium (column), labellum (lip), leaves, pollinia, sepals, stems, roots and the white part of roots of two-year-old, field nursery-grown $D$. officinale plants was determined. Results indicate that all DoC5-MTase and DodMTase genes could be determined in the tested organs, but significant differential expression patterns were observed. According to hierarchical clustering (Fig. 8b), the transcript abundance of DoC5-MTase and DodMTase genes could be broadly split into two groups, the low- and high-expression groups. Members of the low-expression group (DoCMT1, DoCMT2, DoCMT3, DoROS1a and DoROS1b) generally maintained a relatively low level of expression in most tissues with a
FPKM value ranging from 0.64 to 9.36 . However, multiple genes were highly expressed in specific organs, such as DoCMT1 in flower buds and the white part of roots, DoCMT2 in pollinia, and DoROS1a in flower buds. Additionally, members of the high-expression group (DoDRM1, DoDRM2, DoDRM3, DoMET1, DoDML3 and DoDNMT2) were expressed at high levels in all 10 organs with a FPKM value ranging from 12.55 to 47.47 , especially DoDRM2 and DoDML3, suggesting that these genes might play essential roles in plant growth and development. Furthermore, a total of $81.82 \%$ (9/11) of the DoC5-MTase and DodMTase genes showed intermediate and high expression levels in flower buds, pollinia and the white part of roots, compared with $63.64 \%$ (7/11) in the gynostemium and roots.

\section{Expression patterns of DoC5-MTase and DodMTase genes in response to environmental stresses}

D. officinale is an epiphytic orchid that takes root on trunks and cliffs, and is usually exposed to diverse environmental stresses, especially drought and cold stress. To investigate the responses of DoC5-MTase and DodMTase genes under cold stress, $D$. officinale RNA-seq data [37] subjected to ambient $\left(\mathrm{CK}, 20^{\circ} \mathrm{C}\right)$ and cold (CA,

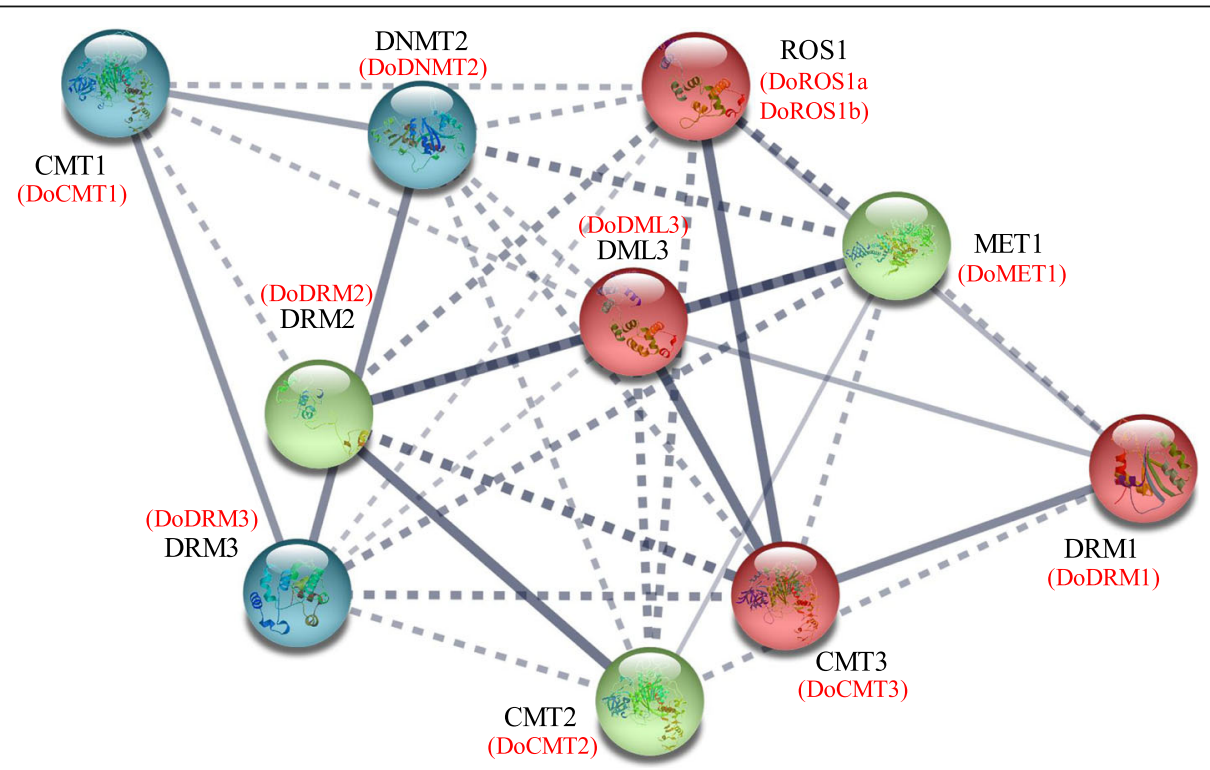

Fig. 6 Potential protein-protein interaction network of DoC5-MTases and DodMTases. The protein interactions are weighted by a correlation coefficient. The dotted lines represent a relatively weak relationship while the solid lines indicate a relatively strong relationship, and the thicker lines indicate stronger correlations 


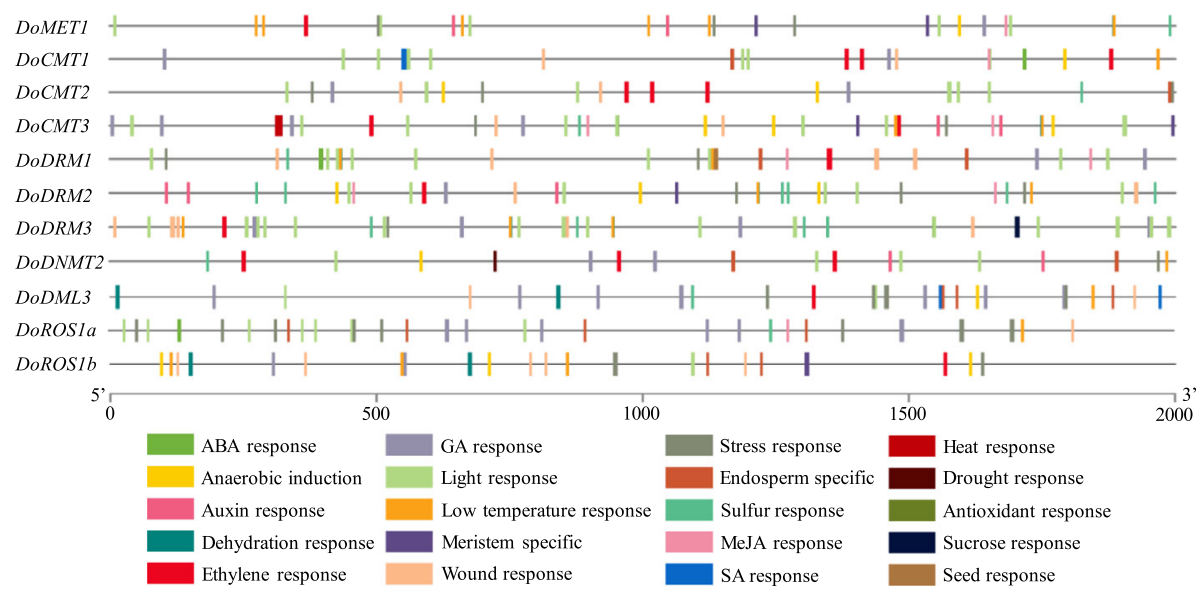

Fig. 7 Prediction of cis-elements in the 2000-bp upstream regulatory regions of DoC5-MTase and DodMTase genes. Different cis-responsive elements are represented by different colored boxes

$0{ }^{\circ} \mathrm{C}$ ) treatment for $20 \mathrm{~h}$ was analyzed. Cold treatment differentially regulated the expression of DoC5-MTase and DodMTase genes (Fig. 9). Compared to the nonacclimated controls, transcript levels of DoCMT3, DoDRM1, DoMET1 and DoDRM2 were suppressed between 1.12- and 2.48-fold. In contrast, DoDML3, DoDNMT2, DoROS1a, DoDRM3, DoCMT1, DoCMT2 and DoROS1b were enhanced between 1.14- and 7.55fold, the highest for DoCMT1 and the lowest for DoDRM3, on average being enhanced 3.79-fold.

To explore the potential roles of DoC5-MTase and DodMTase genes exposed to drought stress, their transcript abundance was evaluated by analyzing the RNA-seq data [32] under different drought treatments. As shown in Fig. 10, DoMET1 was downregulated at both dawn (-1.04-fold) and dusk (-1.32-fold) during drought for 7 days. DoDRM3 was also downregulated at both dawn (-1.14-fold) and dusk (-1.05-fold) during drought for 7 days, but the down-regulation (without a fold-change $\leq-1.2$ ) was not significant. Transcript levels of DoDML3 and DoROS1b remained high (1.56- to 52.36-fold compared to other genes) throughout 7 days of drought stress. Over time, as drought stress increased, the expression of DoDRM1, DoDRM2 and DoCMT3 was notably
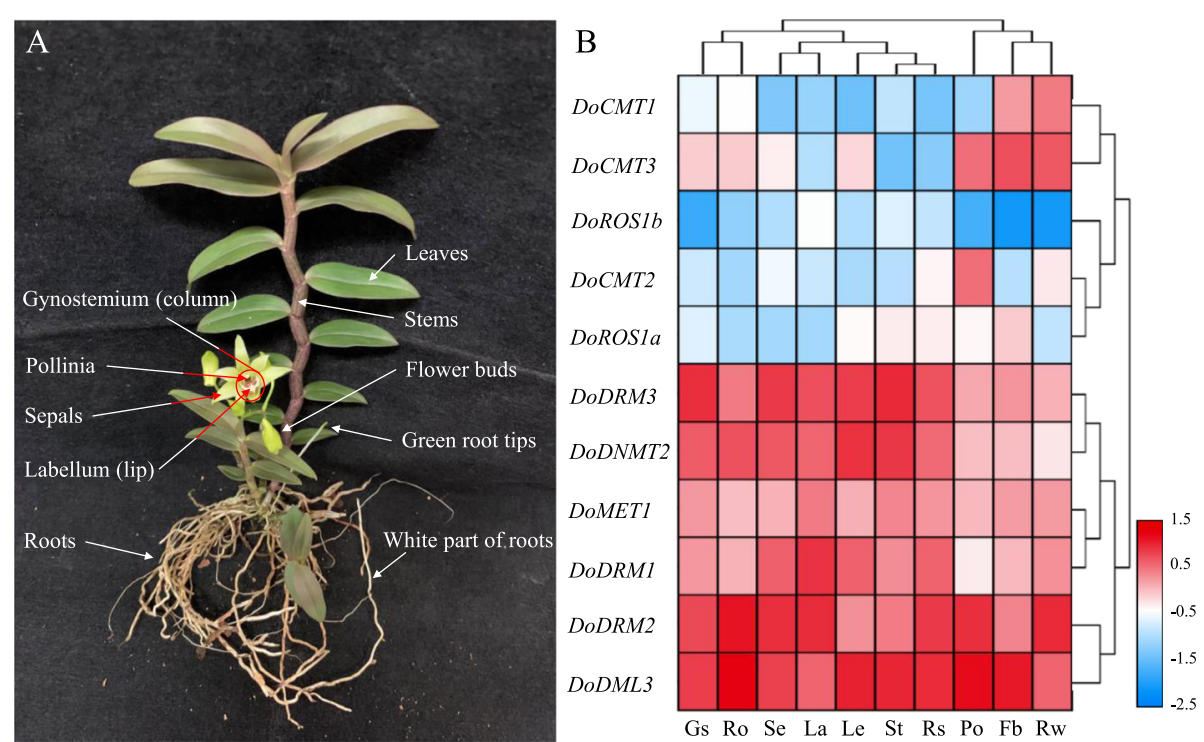

Fig. 8 Tempo-spatial expression patterns of DoC5-MTase and DodMTase genes in different organs in D. officinale. a Different D. officinale organs were used in this study. $\mathbf{b}$ Transcript levels of DoC5-MTase and DodMTase genes in ten D. officinale organs. The log2-transformation of the average of expression values were used to generate the heat map with TBtools software. Blue and red in the color scale indicate low and high transcript expression, respectively. Fb, flower buds; Gs, gynostemium (column); La, labellum (lip); Le, leaves; Po, pollinia; Ro, roots; Rt, green root tips; Rw, white part of roots; Se, sepals; St, stems 

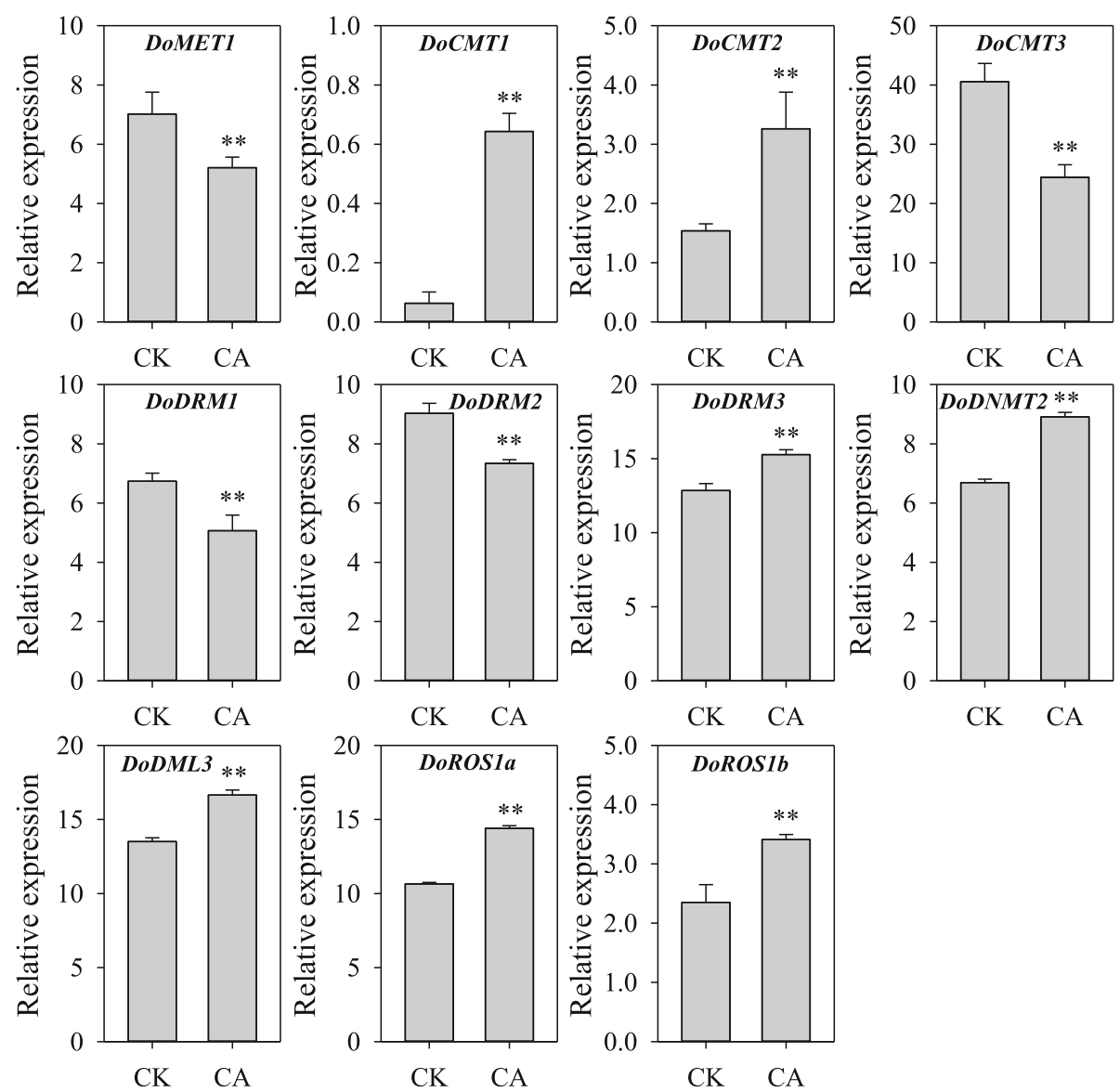

Fig. 9 Transcript levels of DoC5-MTase and DodMTase genes in response to cold stress. Relative expression was quantified by previous D. officinale RNA-seq data [34] subjected to ambient $\left(\mathrm{CK}, 20^{\circ} \mathrm{C}\right)$ and cold $\left(\mathrm{CA}, 0^{\circ} \mathrm{C}\right)$ treatment for $20 \mathrm{~h}$. Error bars indicate the mean \pm SD (standard deviation) of three individual experiments, and were quantified with three independent replicates. Asterisks above bars indicate a significant difference between CK and CA at $p<0.01$ based on the Student's t-test. CA, cold treatment at $0^{\circ} \mathrm{C} ; \mathrm{CK}$, the control at $20^{\circ} \mathrm{C}$

enhanced at dawn (1.93-, 1.05- and 1.01-fold, respectively) throughout 7 days of drought stress, and their expression also improved at dusk (12.41-, 1.14- and 2.45-fold, respectively). Conversely, DoCMT1, DoCMT2, DoROS1a, DoROS1b and DoDNMT2 were downregulated at dawn $(-1.45,-1.71,-1.24$, -2.66 , and -1.26 -fold, respectively) during drought for 7 days, and their expression was also repressed at dusk $(-1.88,-1.23$, $-1.56,-1.58$, and -1.13 -fold, respectively). The expression of DoDNMT2 was upregulated 1.42-fold after the rewatering treatment. Overall, the expression levels of DoC5-MTase and DodMTase genes were significantly influenced by cold and drought stress.

Transcription levels of DoC5-MTase and DodMTase genes during the accumulation of $D$. officinale polysaccharides $D$. officinale is a precious Chinese herb with abundant secondary metabolites, especially WSPs, which account for $34.06-43.27 \% \mathrm{w} / \mathrm{w}$ [28], in its stems. Furthermore, WCPs in D. officinale accumulated at different developmental periods. To elucidate the potential roles of
DoC5-MTase and DodMTase genes involved in the biosynthesis of WSPs, the transcription levels of these genes were assessed across three developmental periods (Fig. 11a). The expression of DodMTase genes (DoDML3, DoROS1a and DoROS1b) was significantly upregulated during a range of plant growth stages (from protocorm-like bodies to plantlets), corresponding to a gradual increase in WSP content that ranged from 93.71 to $183.74 \mathrm{mg} \mathrm{g}^{-1}$ (Figure S11D), as well as the upregulation of six key genes (DoPMM, DoGMP1, DoUGP, DoUGE, DoGMT1 and DoCSLA6) involved in the biosynthesis of WSPs (Fig. 11c). In contrast, in the same stages, the transcript levels of all DoC5-MTase genes (DoMET1, DoCMT1, DoCMT2, DoCMT3, DoDRM1, DoDRM2, DoDRM3 and DoDNMT2) were markedly reduced. Furthermore, the transcript levels of DodMTase genes (DoDML3, DoROS1a and DoROS1b) were positively correlated with WSP content (Pearson's correlation coefficient $R^{2}=0.57,0.87$ and 0.79 , respectively) during three developmental stages (protocorm-like bodies, 


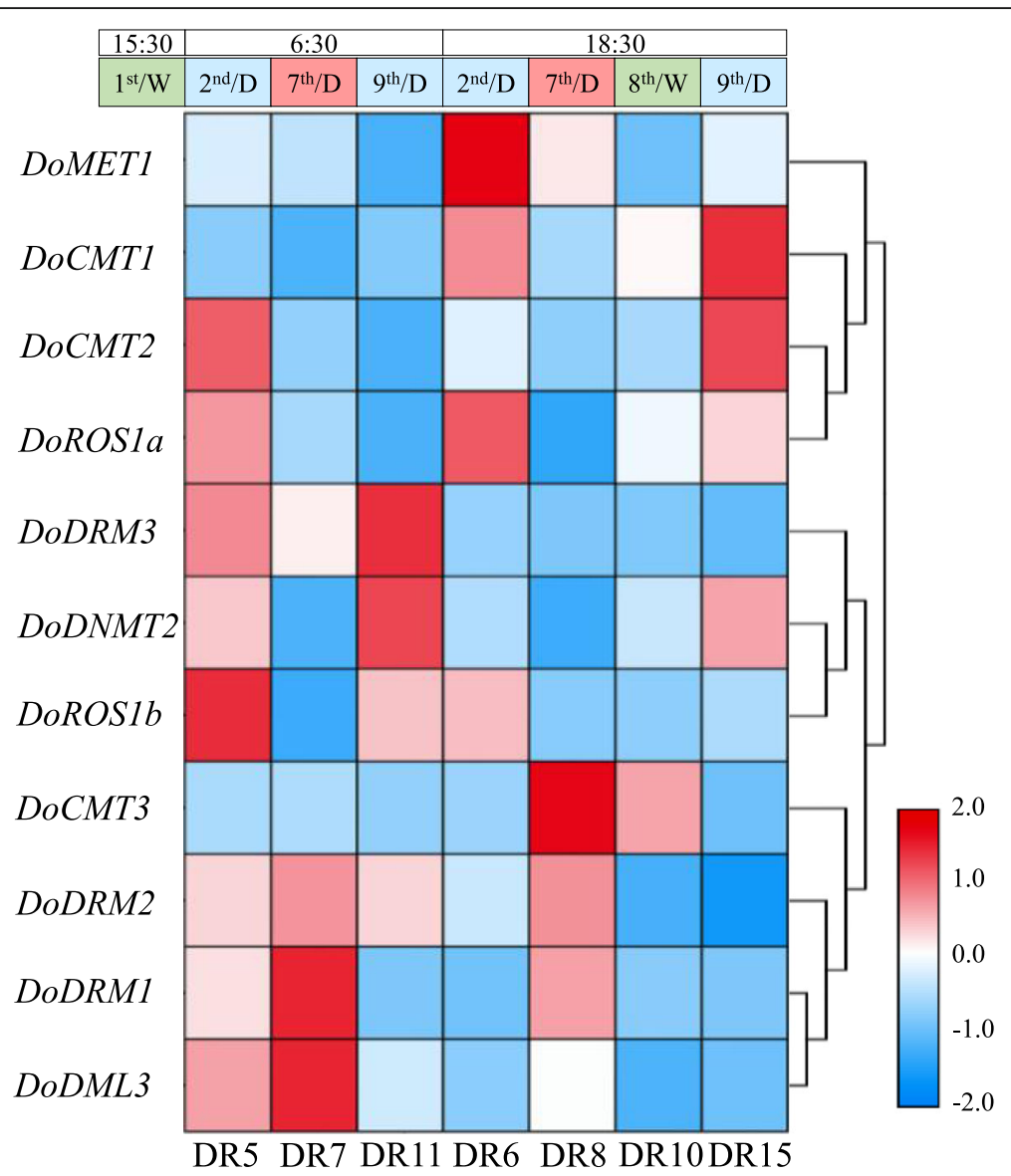

Fig. 10 Transcript levels of DoC5-MTase and DodMTase genes in response to drought stress. Heat map was generated by log2 transformation of the average of the expression values of D. officinale DoC5-MTase and DodMTase genes subjected to drought treatment. D. officinale seedlings were irrigated on the 1st day, dried from the 2nd to the 7th day, and re-watered on the 8th day as shown in Fig. S10. Samples were collected at different times, DR5/DR6, DR7/DR8, and DR11/DR15 were picked at 06:30 and 18:30 on the 2nd, 7th and 9th day, respectively, and DR10 was harvested at 18:30 on the 8th day. Blue and red in the color scale indicate low and high transcript expression, respectively

shoots and plantlets). In contrast, the expression of $D o C 5-$ MTase genes (DoMET1, DoCMT1, DoCMT2, DoCMT3, DoDRM1, DoDRM2, DoDRM3 and DoDNMT2) were negatively correlated with WSP content (Pearson's correlation coefficient $R^{2}=-0.94,-0.95,-0.83,-0.92,-0.66$, $-0.90,-0.96$, and -0.86 , respectively). These findings suggest that DoC5-MTase and DodMTase genes together play roles in the accumulation of WSPs.

\section{Discussion}

D. officinale is a traditional Chinese herb that has been used extensively as a tonic and precious food for thousands of years, due to its abundant bioactive constituents, such as polysaccharides, alkaloids, flavones, and amino acids [27, 38]. Many genes involved in the biosynthesis of bioactive secondary metabolites have been discovered and identified in this orchid. However, no information is available about epigenetic factors (DNA methylation and demethylation) regulating gene expression during the formation of secondary metabolites in $D$. officinale. To elucidate the regulatory role of DNA methylation and demethylation in D. officinale, we identified the DoC5-MTase and DodMTase genes at the whole-genome level. The cis-acting elements, conserved motifs, phylogenetic relationships, protein-protein interactions, and sequence features of these genes were also integratively analyzed.

According to the D. officinale reference genome [33, 34], a total of eight DoC5-MTase and three DodMTase genes were discovered and identified (Table 1). However, the number of C5-MTase and dMTase gene families mined in A. thaliana (15), globe artichoke (Cynara cardunculus) (17) and S. miltiorrhiza (14) is higher than that of D. officinale (11). Since the $D$. officinale genome $(1.35 \mathrm{~GB})$ is approximately 2- to 11 -fold larger than that of A. thaliana (125 MB) [39], globe artichoke (725 MB) [40] and S. miltiorrhiza (558 MB) [41], this suggests that DoC5-MTase and DodMTase may have gene absence or mutation events in D. officinale. Moreover, A. thaliana contains four MET genes, while $D$. officinale has only one (Figs. 4,5), indicating the 

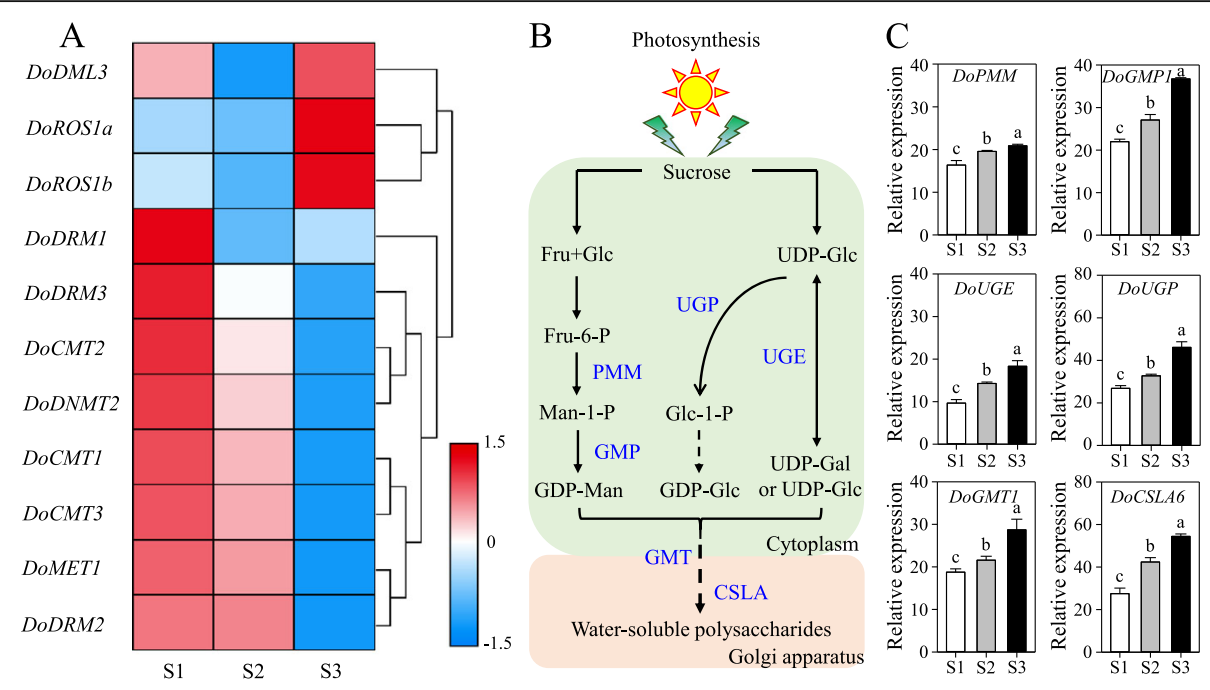

Fig. 11 Transcript levels of DoC5-MTase and DodMTase genes, as well as the genes involved in the biosynthesis of WSPs, at different developmental stages. a The expression patterns of DoC5-MTase and DodMTase genes during different developmental stages. b Proposed biosynthetic pathway of WSPs in D. officinale. $\mathbf{c}$ The expression patterns of genes involved in the biosynthesis of WSPs during different developmental stages. The three developmental stages are protocorm-like bodies (S1), shoots (S2) and plantlets (derived from protocorm-like bodies, S3), which were collected at 2, 4 and 10 months after germination, respectively. The heat map was generated by log2 transformation of the average of the expression values of D. officinale DoC5-MTase and DodMTase genes during different developmental stages. Blue and red in the color scale indicate low and high transcript expression, respectively. In C, error bars indicate the mean \pm SD (standard deviation) of three individual experiments, and were quantified with three independent replicates. Different lowercase letters above bars indicate a significant difference among different stages at $p<0.05$ based on Duncan's multiple range test. CSLA, cellulose synthase-like A; Fru, fructose; Gal, galactose; Glc, glucose; GMP, GDP-mannose pyrophosphorylase; Man, mannose; PMM, phosphomannomutase; UGE, UDP-glucose epimerase; UGP, UDPglucose pyrophosphorylase; WSP, water-soluble polysaccharide

loss of MET genes during the evolution of $D$. officinale. Similarly, no DME was found in O. sativa or D. officinale, unlike in A. thaliana (Figs. 4, 5). Although ROS1 was found in D. officinale, it contains two paralogs, DoROS1a and DoROS1b (Figs. 4, 5), inferring the occurrence of ROS1 gene duplication. In previous studies, 756 gene families expanded, whereas 804 gene families contracted during the evolution of $D$. officinale [34]. The methylation-related genes are associated with whole genome duplication and tandem replication, along with gene absence events [42, 43], thus resulting in the differential gene number between $D$. officinale and other plant species, such as A. thaliana, globe artichoke and S. miltiorrhiza. Gene duplication and loss events play a pivotal role in the evolution of DoC5-MTase and DodMTase gene families, which is in agreement with research in Fragaria vesca [44] and tea plant [26]. Taken together, the duplication of DoROS1 and the loss of DoDME and DoMET1 implied that DoC5-MTase and DodMTase might have functional redundancy and divergence.

Phylogenetic analysis illustrated that DoC5-MTase was divided into four classes, including MET, CMT, DRM and DNMT (Figs. 3, 4, 5), which are highly conserved in both dicots and monocots, such as A. thaliana [21] and O. sativa [45]. DodMTase was grouped into two categories, DML3 and ROS1 (Fig. 2), which is inconsistent with the result of dicots, such as A. thaliana [21] and peanut
(Arachis hypogaea) [22], but is consistent with the results of monocots, such as rice and maize [46]. This suggests that DME may be phylogenetically monophyletic in dicots. Furthermore, each C5-MTase or dMTase subfamily was further divided into dicot and monocot groups (Fig. 5). These results were verified by MEME analysis, and the conserved motifs were similar within each sub-cluster, but divergent among different clusters (Fig. 5), indicating that dicots and monocots may adopt different tactics in the evolution of C5-MTases and dMTases.

Within a gene family, genes harboring the same domains and motifs may perform similar roles. Cytosine- 5 DNA methylation, including de novo and maintenance DNA methylation, is antagonistically controlled by C5MTases and dMTases. CMT, DRM, and MET are responsible for the maintenance of $\mathrm{CHG}, \mathrm{CHH}$, and $\mathrm{CG}$ methylation, respectively, while DRM is the main de novo C5-MTase [1, 2, 4]. DNMT2 exhibits transfer RNA methyltransferase activity [47], but its role in cytosine-5 DNA methylation is still unknown. DNA methylation plays a critical role in growth and development. The drm1drm2cmt3 triple mutation leads to a dwarf phenotype, partial sterility, and slow growth in A. thaliana [48]. DNA methylation is also implicated in the regulation of fruit ripening. Genes involved in RNA-directed 
DNA methylation, including FvCMT3.1, FvCMT3.2, FvDRM1.3, and FvDRM3.1, are downregulated and thus lead to DNA hypomethylation during strawberry ripening [49]. For DNA demethylase, ROS1 dysfunction can silence the expression of the $R D 29 A$ gene, because of its hypermethylation at the $R D 29 A$ promoter [50]. DME activates maternal FIS2, FWA, and MEA allele expression, and is responsible for endosperm gene imprinting and seed viability [51]. SIDML2, a ROS1 ortholog, is required for tomato fruit ripening, by activating DNA demethylation and promoting the expression of fruit-ripening genes, such as CNR, PSY1, and RIN [13]. OsROS1a plays an irreplaceable role in both male and female gametophytes [52], while OsROS1c enhances expression and transposition of Tos17 in rice callus [53]. This suggests that C5-MTase and dMTase genes from a cluster might also participate in distinct biological processes.

Increasing evidence shows that C5-MTases and $\mathrm{dMTases}$ are involved in abiotic stress responses [7-9]. In the present study, a large amount of hormone-, light-, and stress-responsive cis-acting elements was detected in the promoter regions of DoC5-MTase and DodMTase genes (Fig. 7). Besides, cis-acting elements function as molecular switches, participating in the transcriptional regulation of stress-inducible gene expression and regulating various biological processes [54]. In addition, abiotic stress can cause variation in cytosine DNA methylation by generating novel epialleles, potentially affecting transcript abundance [55]. This effect may account for the significant changes in the transcript abundance of DoC5-MTase and DodMTase under cold stress (Fig. 9), and drought stress (Fig. 10). Consistent with a previous study [56], cis-acting elements harboring stress-inducible roles in DoC5-MTase and DodMTase, may respond to multiple stress signals and affect gene expression.

Notably, DNA methylation and demethylation are involved in the regulation of secondary metabolism. WSPs are important secondary metabolites and account for the commercial quality of D. officinale [33]. The WSP content in different parts of $D$. officinale differs (stem > leaves > roots) [38]. Herein, DoC5-MTase and DodMTase genes exhibited significantly differential transcription levels in the flower bud, gynostemium, labellum, leaf, pollinium, root, sepal, and stem (Fig. 8). The trend of DoROS1a expression is consistent with that of the WSP content, whereas the trend of DoCMT3 expression was contrary to the above, indicating that DoC5-MTase and DodMTase are associated with the production of WSPs. In addition, cold stress can markedly improve WSP content in D. officinale [31, 37]. Interestingly, three DodMTase genes was upregulated, and eight DoC5MTase genes were downregulated (Fig. 9) either in response to cold stress or during seedling development
(Fig. 11). DNA methylation inhibits gene transcription. In contrast, DNA demethylation enhances gene transcription. Cold treatment may activate the enzymes involved in the biosynthesis of WSPs through increased DNA demethylation and decreased DNA methylation. Taken together, DoC5-MTase and DodMTase may work together, affecting the accumulation of WSPs by regulating the level of DNA methylation, but the mechanisms need to be studied in greater detail.

\section{Conclusions}

According to the $D$. officinale reference genome, eight DoC5-MTase genes and three DodMTase genes were discovered and identified. Phylogenetic analysis demonstrated that the eight DoC5-MTase genes were divided into four categories, DoCMT, DoDRM, DoMET, and DoDNMT. The three DodMTase genes were grouped into DoROS and DoDML subfamilies. The expression profiles of DoC5-MTase and DodMTase genes suggest their functional importance in the accumulation of WSPs and response to stresses in D. officinale. These findings will be helpful to reveal the possible roles of DoC5-MTase and DodMTase in secondary metabolism and stress responses in D. officinale, and provide constructive clues for further exploration of the epigenetic mechanism of DoC5-MTase and DodMTase in orchids.

\section{Methods}

\section{Plant material and experimental treatments}

D. officinale "Zhongke 1" (a high-WSP line, $49.5 \% \mathrm{w} / \mathrm{w}$; genetic breeding by Prof. Jun Duan at the South China Botanical Garden, Chinese Academy of Sciences, Approval no. 20180003, website: https://www.cas.cn/syky/2 01810/t20181031_4668440.shtml) plants were cultivated and acclimatized in a walk-in phytotron at the South China Botanical Garden, Chinese Academy of Sciences, Guangzhou, in China. Tissue-specific mRNA expression patterns of DoC5-MTase and DodMTase genes were assessed for protocorm-like bodies, shoots and plantlets (derived from protocorm-like bodies) during the vegetative stage $(2,4$ and 10 months after $D$. officinale seeds were germinated on half-strength Murashige and Skoog medium [57], respectively; Figure S10A, B, C), as well as 10 tissues, including flower buds, gynostemium (column), sepals, labellum (lip), leaves, roots, stems, pollinia, white part of roots and green root tips at the reproductive stage (14 months after seedlings were transplanted into plastic pots; Fig. 8a). Four-month-old seedlings were placed in controlled-climate chambers with a 12-h light/ 12-h dark cycle, $80 \%$ relative humidity, and $60 \mu \mathrm{mol}$ $\mathrm{m}^{-2} \mathrm{~s}^{-1}$ photosynthetically active radiation at $0{ }^{\circ} \mathrm{C}$ (for cold treatment) and $20^{\circ} \mathrm{C}$ (for ambient control) for $20 \mathrm{~h}$, respectively. All sampled materials were free of any insects, disease or mechanical damage. Each treatment was 
conducted as three replications and with at least 10 independent plants per condition. All these samples were immediately stored at $-80^{\circ} \mathrm{C}$ after freezing in liquid nitrogen.

\section{Identification of the cytosine-5 DNA methyltransferase and demethylase genes}

The $D$. officinale genome was retrieved from the online Herbal Medicine Omics Database (http://herbalplant. ynau.edu.cn/) [33], or downloaded from DDBJ/EMBL/ GenBank under the accession code SUB764497 [34]. The Hidden Markov model (HMM) of cytosine-5specific DNA-methylase (PF00145), was obtained from the PFAM database (http://pfam.xfam.org/) and employed C5-MTase proteins with HMMER software version 3.2.1 (http://hmmer.org/). Similarly, to search the dMTase proteins, the HMMs of the helix-hairpinhelix, the Gly/Pro-rich loop (HhH-GPD, PF00730) and RNA recognition motif demethylase (RRM-DME, PF15628) were downloaded from the PFAM database as the probes. Afterwards, C5-MTase and dMTase proteins from $D$. officinale were verified via a local HMM-based search program $\left(E-v a l u e ~ \leq 1 \mathrm{e}^{-10}\right)$. Subsequently, these candidate proteins, annotated as "cytosine- 5 DNA methyltransferase" or "DNA demethylase" with the best "C5MTase" or "HhH-GPD and RRM-DME" hits, were retained for further confirmation with the Conserved Domain Database (NCBI-CDD, https://www.ncbi.nlm.nih. gov/cdd) and the Simple Modular Architecture Research Tool (SMART, http://smart.embl-heidelberg.de/). Redundant sequences and incomplete proteins containing no characteristic PF00145, PF00730 or PF15628 motifs were manually removed. All putative methyltransferase and demethylase genes that were identified are listed in Table S1. The grand average of hydrophobicity (GRAVY), molecular weight (MW) and isoelectric point (pI) of DoC5MTase and DodMTase proteins were calculated using the ExPASy tool (http://web.expasy.org/protparam). PlantmPLoc (http://www.csbio.sjtu.edu.cn/bioinf/plant-multi/) was used to predict the sub-cellular localization of DoC5MTase and DodMTase genes.

\section{Phylogenetic tree construction}

DNAMAN version 6.0 software (Lynnon Biosoft, Foster City, CA, USA) was used to generate multiple sequence alignments of the C5-MTase and dMTase proteins among different plant species including A. lyrata, A. thaliana, Brachypodium distachyon, Citrus clementina, Cucumis sativus, Erythranthe guttata, Glycine max, Manihot esculenta, Oryza sativa, Populus trichocarpa, $R$. communis, S. lycopersicum, Salvia miltiorrhiza, Sorghum bicolor, Zea mays and Vitis vinifera, that were retrieved from Phytozome version 12.1.6 (https://phytozome.jgi. doe.gov/pz/portal.html\#). To verify the sequence alignments using DNAMAN, multiple alignment of the C5-MTase and dMTase proteins was also performed using the ClustalX 2.1 program [58]. The resulting file was used to build a phylogenetic tree with the Molecular Evolutionary Genetics Analysis (MEGA X) software [59] based on the neighbor-joining method [60] with 1000 bootstrap replicates. DoC5-MTase and DodMTase were classified based on their phylogenetic relationship with the corresponding A. thaliana C5-MTase and dMTase proteins.

Analysis of conserved motifs, gene structure and proteinprotein interaction

Conserved motifs of all DoC5-MTase and DodMTase proteins were analyzed using Multiple Expectation Maximization for Motif Elicitation (MEME, http:// meme-suite.org/) software version 5.0 .5 by uploading the amino acid sequences following the MEME instructions. All coding sequences and genomic sequences of DoC5MTase and DodMTase genes were used as individual queries to visualize the schematic diagrams of the genes' structures using Gene Structure Display Server version 2.0 (GSDS, http://gsds.cbi.pku.edu.cn/). The STRING 11 tool (https://string-db.org) was used to construct a protein-protein interaction network.

\section{RNA preparation and RT-qPCR analysis}

Total RNA was extracted from the flowers, leaves, roots and stems of 14-month-old D. officinale after transplantation by RNAout2.0 reagent (Tiandz Inc., Beijing, China) as described previously [25]. RNA was purified with RNase-free DNase I (Takara Bio Inc., Kyoto, Japan). Firststrand cDNA was synthesized from $1 \mu \mathrm{g}$ of total RNA using the PrimeScript Reagent Kit with gDNA Eraser (Takara Bio Inc.) according to the supplier's instructions. The resulting CDNA was diluted 1:50 and used as a template for RT-qPCR analysis. RT-qPCR was performed on a LightCycler 480 instrument (Roche Diagnostics, Mannheim, Germany) on 96-well plates with iTaq ${ }^{\mathrm{Tm}}$ Universal SYBR $^{\circ}$ Green Supermix (Bio-Rad Laboratories, Hercules, CA, USA) with an identified amplification program for 40 cycles, as described previously [28]. The constitutively expressed D. officinale ACTIN (NCBI accession number: JX294908) was used as the internal standard for normalizing variations in cDNA concentration. In addition, at least three biological replicates derived from at least three independently generated cDNA samples were analyzed. The specific primer sequences of the C5-MTase and dMTase genes are listed in Table S1.

\section{Transcriptome analysis of DoC5-MTase and DodMTase gene expression}

The expression profiles of DoC5-MTase and DodMTase genes under ambient $\left(20^{\circ} \mathrm{C}\right)$ and cold $\left(0^{\circ} \mathrm{C}\right)$ for $20 \mathrm{~h}$ were 
investigated [34]. RNA-seq reads were obtained from the NCBI sequence read archive (SRA) database under accession numbers SRR3210613, SRR3210621, SRR3210626, SRR3210630, SRR3210635 and SRR3210636. To detect the transcription abundance of DoC5-MTase and DodMTase genes subjected to drought and re-watering treatments, $D$. officinale seedlings were watered on the 1st day, and kept unwatered to mimic natural drought stress from the 2nd to the 7th day, and rewatered on the 8th day. Samples were individually collected at both $06: 30$ and 18 : 30 on the 2nd (DR5 and DR6), 7th (DR7 and DR8), and 9th (DR11 and DR15) day, and at 18:30 on the 8th day (DR10). The RNA-seq data of DR5 (SRR7223299), DR6 (SRR7223298), DR7 (SRR7223301), DR8 (SRR7223300), DR10 (SRR7223296), DR11 (SRR7223295) and DR15 (SRR7223297) were downloaded from the NCBI SRA provided by Wan et al. [32]. All clean reads were aligned to the $D$. officinale reference genome [33, 34] using HISAT2 software [61] with defaults parameters. After the final transcriptome was established, StringTie software [62] was used to estimate the differential transcript levels for mRNAs by quantifying the FPKM value. The expression levels of all transcripts were indicated by calculating fragments per kilobase per million (FPKM). The differentially expressed DoC5-MTase and DodMTase genes were defined with $\log 2$ (fold change) $>1$ or $<-1$ and with statistical significance $(p<0.05)$. Furthermore, the targeted genes with a fold-change $\geq 1.2$ were defined as upregulated genes, and those with a fold-change $\leq-1.2$ were regarded as down-regulated genes.

\section{Determination of WSP content in D. officinale during three developmental periods}

The WSP content was quantified using the phenolsulfuric acid method as described previously [31]. Briefly, powdered samples $(0.3 \mathrm{~g})$ of $D$. officinale protocorm-like bodies, shoots and plantlets was pre-extracted with aqueous-ethanol solution $(80 \%, v / v)$ at $80^{\circ} \mathrm{C}$ for $2 \mathrm{~h}$, to remove small molecular weight compounds. The residue was re-extracted with $100 \mathrm{~mL}$ of distilled water at $100^{\circ} \mathrm{C}$ for $2 \mathrm{~h}$, then filtered through Whatman No. 1 filter paper (Congyuan Instrument Co., Guangzhou, China). The filtrate was used to assess the WSP content with a phenol-sulfuric acid method based on a glucose standard curve at $488 \mathrm{~nm}$ on a UV-6000 spectrophotometer (Shanghai Metash Instruments Co., Shanghai, China).

\section{Statistical analysis}

All data were expressed as the mean \pm standard error of more than three independent biological replicates for each determination. Statistical analysis was performed using IBM SPSS version 22.0 for Windows (IBM Corp., Armonk, NY, USA). The significant difference of transcript levels between CK and CA at $p<0.01$ was analyzed based on the Student's $t$-test. Unless otherwise indicated, all data were compared by using analysis of variance (ANOVA) followed by Duncan's multiple range test at $p$ $<0.05$. Heat maps were generated by the software TBtools (https://github.com/CJ-Chen/TBtools), and their color scales represent the log2-transformation of the average of expression values, with low expression in blue and high expression in red. Correlation analysis was performed using Pearson's correlation coefficient $\left(R^{2}\right)$ at $p<0.01$.

\section{Supplementary Information}

The online version contains supplementary material available at https://doi. org/10.1186/s12870-020-02811-8.

Additional file 1: Table S1. Primers used for RT-qPCR analysis in this study. Gene-specific primers for real-time reverse transcription quantitative PCR (RT-qPCR) were designed by the PrimerQuest tool (http://www. idtdna.com/Primerquest/Home/Index). The D. officinale ACTIN gene was obtained from NCBI (GenBank accession no. JX294908). F, forward; R, reverse

Additional file 2: Table S2. Distribution of conserved motifs in DoC5MTase based on the results of MEME (http://meme-suite.org/) analysis

Additional file 3: Table S3. Distribution of conserved motifs in DodMTase based on the results of MEME (http://meme-suite.org/) analysis

Additional file 4: Table S4. Information of C5-MTase genes in the 11 tested species

Additional file 5: Table S5. Information of dMTase genes in the 16 tested species

Additional file 6: Table S6. Number of cis-elements in the promoter region of DoMTase and dMTase genes

Additional file 7: Figure S1. Sequence alignment of MET1 protein sequences from $D$. officinale and $A$. thaliana

Additional file 8: Figure S2. Sequence alignment of CMT protein sequences from $D$. officinale and $A$. thaliana

Additional file 9: Figure S3. Sequence alignment of DRM protein sequences from $D$. officinale and $A$. thaliana

Additional file 10: Figure S4. Sequence alignment of DNMT2 protein sequences from $D$. officinale and $A$. thaliana

Additional file 11: Figure S5. Sequence alignment of DML3 protein sequences from $D$. officinale and $A$. thaliana

Additional file 12: Figure S6. Sequence alignment of ROS1 protein sequences from $D$. officinale and $A$. thaliana

Additional file 13: Figure S7. Distribution of conserved motifs in DoC5-MTase based on the results of MEME analysis

Additional file 14: Figure S8. Distribution of conserved motifs in DodMTase based on the results of MEME analysis

Additional file 15: Figure S9. Number of cis-elements in the promoter region of DoC5-MTase and DodMTase genes

Additional file 16: Figure S10. The experimental scheme of $D$. officinale seedlings under sustained drought treatment and irrigation. Green arrows at 15:30 indicate the irrigation times, and magenta arrows indicate the experimental sampling times. White bars indicate light periods, and black bars indicate dark periods

Additional file 17: Figure S11. WSP content in juvenile D. officinale stems at three different developmental stages (protocorm-like bodies, shoots and plantlets). (A-C) Three developmental stages (protocorm-like bodies, shoots and plantlets, namely S1, S2 and S3, respectively), which correspond to 2, 4 and 10 months after germination, respectively. (D) WSP content in protocorm-like bodies, shoots and plantlets of juvenile D. officinale stems. Error bars indicate the mean \pm SD (standard deviation) of 
three individual experiments, and were performed in triplicate. Differen letters above bars indicate a significant difference among different stages at $p<0.05$ based on Duncan's multiple range test. DW, dry weight. WSP, water-soluble polysaccharide

\section{Abbreviations}

C5-MTase: Cytosine-5 DNA methyltransferase; CMT3: CHROMOMETHYLASE3; DME: DEMETER; DML2: DEMETER-like 2; DML3: DEMETER-like 3; dMTase: DNA demethylase; DRM2: DOMAINS REARRANGED METHYLASE2; FPKM: Fragments per kilobase per million; GRAVY: Grand average of hydrophobicity; HMM: Hidden Markov model; MEGA: Molecular evolutionary genetics analysis; MEME: Multiple expectation maximization for motif elicitation; MET1: METHYLTRANSFERASE1; MW: Molecular weight; ORF: Open reading frame: pl: isoelectric point: ROS1: REPRESSOR OF SILENCING1: RTqPCR: Reverse transcription quantitative polymerase chain reaction; WSP: Water-soluble polysaccharide

\section{Acknowledgements}

We thank all the colleagues in our laboratory for help, advice, and discussion. We thank the editor and reviewers for evaluating and providing constructive comments on this manuscript.

\section{Authors' contributions}

JD and JATdS supervised the project. ZY, JATdS and JD conceived the research program and designed research. ZY, GZ, CZ, CH, CS and MZ performed research, ML performed the bioinformatics analyses, $Z Y$ and JATdS analyzed the data. ZY wrote the manuscript, ZY and JATdS revised this manuscript. All authors have read and agreed to the published version of the manuscript. Corresponding author. Jun Duan. All authors read and approved the final manuscript.

\section{Funding}

This research was supported by the National Natural Science Foundation of China (Grant number 32000257 and 31871547), the Key Area R\&D Project of Guangdong Province (Grant number 2020B020221001) and the National Key Research and Development Program of China (Grant number 2018YFD1000406). The funding agencies were not involved in study design, data collection and analysis, or preparation of the manuscript.

\section{Availability of data and materials}

The datasets supporting the conclusions of this article are included within the article and its additional files. All data and plant materials used in current study are available from the corresponding author on reasonable request.

\section{Ethics approval and consent to participate}

Not applicable.

\section{Consent for publication}

Not applicable.

\section{Competing interests}

The authors declare that they have no competing interests.

\section{Author details}

${ }^{1}$ Key Laboratory of South China Agricultural Plant Molecular Analysis and Genetic Improvement, South China Botanical Garden, Chinese Academy of Sciences, Guangzhou 510650, China. ${ }^{2}$ Economic Botany, Core Botanical Gardens, Chinese Academy of Sciences, Guangzhou 510650, China. ${ }^{3}$ Independent researcher, P. O. Box 7, Miki-cho post office, Ikenobe 3011-2, Miki-cho, Kagawa-ken 761-0799, Japan. ${ }^{4}$ Biodata Biotechnology Co. Ltd, Hefei 230031, China.

\section{Received: 14 May 2020 Accepted: 22 December 2020} Published online: 06 January 2021

\section{References}

1. Zhu JK. Active DNA demethylation mediated by DNA glycosylases. Annu Rev Genet. 2009;43:143-66

2. Zhang H, Lang Z, Zhu JK. Dynamics and function of DNA methylation in plants. Nat Rev Mol Cell Biol. 2018;19:489-506.
3. Liu R, Lang Z. The mechanism and function of active DNA demethylation in plants. J Integr Plant Biol. 2020;62:148-59.

4. Law JA, Jacobsen SE. Establishing, maintaining and modifying DNA methylation patterns in plants and animals. Nat Rev Genet. 2010;11:204-20.

5. Zemach A, Kim MY, Hsieh PH, Coleman-Derr D, Eshed-Williams L, Thao K, Harmer SL, Zilberman D. The Arabidopsis nucleosome remodeler DDM1 allows DNA methyltransferases to access $\mathrm{H1}$-containing heterochromatin. Cell. 2013;153:193-205.

6. Parrilla-Doblas JT, Roldán-Arjona T, Ariza RR, Córdoba-Cañero D. Active DNA demethylation in plants. Int J Mol Sci. 2019;20:E4683.

7. Liu T, Li Y, Duan W, Huang F, Hou X. Cold acclimation alters DNA methylation patterns and confers tolerance to heat and increases growth rate in Brassica rapa. J Exp Bot. 2017;68:1213-24.

8. Ganguly DR, Crisp PA, Eichten SR, Pogson BJ. The Arabidopsis DNA methylome is stable under transgenerational drought stress. Plant Physiol. 2017;175:1893-912.

9. Ma Y, Min L, Wang M, Wang C, Zhao Y, Li Y, Fang Q, Wu Y, Xie S, Ding Y, Su X, Hu Q, Zhang Q, Li X, Zhang X. Disrupted genome methylation in response to high temperature has distinct effects on microspore abortion and anther indehiscence. Plant Cell. 2018;30:1387-403.

10. Chen X, Schönberger B, Menz J, Ludewig U. Plasticity of DNA methylation and gene expression under zinc deficiency in Arabidopsis roots. Plant Cell Physiol. 2018;59:1790-802.

11. Chen R, Li M, Zhang H, Duan L, Sun X, Jiang Q, Zhang H, Hu Z. Continuous salt stress-induced long non-coding RNAs and DNA methylation patterns in soybean roots. BMC Genomics. 2019;20:730

12. Marfil C, Ibañez V, Alonso R, Varela A, Bottini R, Masuelli R, Fontana A, Berli F. Changes in grapevine DNA methylation and polyphenols content induced by solar ultraviolet-B radiation, water deficit and abscisic acid spray treatments. Plant Physiol Biochem. 2019;135:287-94.

13. Lang Z, Wang Y, Tang K, Tang D, Datsenka T, Cheng J, Zhang Y, Handa AK, Zhu JK. Critical roles of DNA demethylation in the activation of ripeninginduced genes and inhibition of ripening-repressed genes in tomato fruit. Proc Natl Acad Sci U S A. 2017:114:E4511-9.

14. Huang H, Liu R, Niu Q, Tang K, Zhang B, Zhang H, Chen K, Zhu JK, Lang Z. Global increase in DNA methylation during orange fruit development and ripening. Proc Natl Acad Sci U S A. 2019;116:1430-6.

15. Zha L, Liu S, Liu J, Jiang C, Yu S, Yuan Y, Yang J, Wang Y, Huang L. DNA methylation influences chlorogenic acid biosynthesis in Lonicera japonica by mediating LjbZIP8 to regulate phenylalanine ammonia-lyase 2 expression. Front Plant Sci. 2017:8:1178.

16. Wang L, Wang C, Liu X, Cheng J, Li S, Zhu JK, Gong Z. Peroxisomal Boxidation regulates histone acetylation and DNA methylation in Arabidopsis. Proc Natl Acad Sci U S A. 2019;116:10576-85.

17. Xiao K, Chen J, He Q, Wang Y, Shen H, Sun L. DNA methylation is involved in the regulation of pepper fruit ripening and interacts with phytohormones. J Exp Bot. 2020;71:1928-42.

18. Ma CQ, Jing CJ, Chang B, Yan JY, Liang BW, Liu L, Yang YZ, Zhao ZY. The effect of promoter methylation on MdMYB1 expression determines the level of anthocyanin accumulation in skins of two non-red apple cultivars. BMC Plant Biol. 2018:18:108.

19. Zhu YC, Zhang B, Allan AC, Lin-Wang K, Zhao Y, Wang K, Chen KS, Xu CJ. DNA demethylation is involved in the regulation of temperature-dependent anthocyanin accumulation in peach. Plant J. 2020;102:965-76.

20. Liu J, Wu X, Yao X, Yu R, Larkin PJ, Liu CM. Mutations in the DNA demethylase OsROS1 result in a thickened aleurone and improved nutritional value in rice grains. Proc Natl Acad Sci U S A. 2018;115: 11327-32.

21. Ogneva ZV, Dubrovina AS, Kiselev KV. Age-associated alterations in DNA methylation and expression of methyltransferase and demethylase genes in Arabidopsis thaliana. Biol Plant. 2016;60:628-34.

22. Wang P, Gao C, Bian X, Zhao S, Zhao C, Xia H, Song H, Hou L, Wan S, Wang $X$. Genome-wide identification and comparative analysis of cytosine-5 DNA methyltransferase and demethylase families in wild and cultivated peanut. Front Plant Sci. 2016;7:7

23. Fan S, Liu H, Liu J, Hua W, Xu S, Li J. Systematic analysis of the DNA methylase and demethylase gene families in rapeseed (Brassica napus L.) and their expression variations after salt and heat stresses. Int J Mol Sci. 2020;21:953.

24. Victoria D, Aliki K, Venetia K, Georgios M, Zoe H. Spatial and temporal expression of cytosine-5 DNA methyltransferase and DNA demethylase 
gene families of the Ricinus communis during seed development and drought stress. Plant Growth Regul. 2018;84:81-94.

25. Cao D, Ju Z, Gao C, Mei X, Fu D, Zhu H, Luo Y, Zhu B. Genome-wide identification of cytosine-5 DNA methyltransferases and demethylases in Solanum Iycopersicum. Gene. 2014;550:230-7.

26. Zhu C, Zhang S, Zhou C, Chen L, Fu H, Li X, Lin Y, Lai Z, Guo Y. Genomewide investigation and transcriptional analysis of cytosine-5 DNA methyltransferase and DNA demethylase gene families in tea plant (Camellia sinensis) under abiotic stress and withering processing. PeerJ. 2020;8:e8432.

27. Teixeira da Silva JA, Ng TB. The medicinal and pharmaceutical importance of Dendrobium species. Appl Microbiol Biotechnol. 2017;101:2227-39.

28. Yu Z, He C, Teixeira da Silva JA, Luo J, Yang Z, Duan J. The GDP-mannose transporter gene (DoGMT) from Dendrobium officinale is critical for mannan biosynthesis in plant growth and development. Plant Sci. 2018;277:43-54.

29. He C, Yu Z, Teixeira da Silva JA, Zhang J, Liu X, Wang X, Zhang X, Zeng S, Wu K, Tan J, Ma G, Luo J, Duan J. DoGMP1 from Dendrobium officinale contributes to mannose content of water-soluble polysaccharides and plays a role in salt stress response. Sci Rep. 2017;7:41010.

30. Yu Z, He C, Teixeira da Silva JA, Zhang G, Dong W, Luo J, Duan J. Molecular cloning and functional analysis of DoUGE related to water-soluble polysaccharides from Dendrobium officinale with enhanced abiotic stress tolerance. Plant Cell Tissue Organ Cult. 2017;131:579-99.

31. Yu Z, Yang Z, Teixeira da Silva JA, Luo J, Duan J. Influence of low temperature on physiology and bioactivity of postharvest Dendrobium officinale stems. Postharvest Biol Technol. 2019;148:97-106.

32. Zou LH, Wan X, Deng H, Zheng BQ, Li BJ, Wang Y. RNA-seq transcriptomic profiling of crassulacean acid metabolism pathway in Dendrobium catenatum. Sci Data. 2018:5:180252.

33. Yan L, Wang X, Liu H, Tian Y, Lian J, Yang R, Hao S, Wang X, Yang S, Li Q, Qi S, Kui L, Okpekum M, Ma X, Zhang J, Ding Z, Zhang G, Wang W, Dong Y, Sheng J. The genome of Dendrobium officinale illuminates the biology of the important traditional Chinese orchid herb. Mol Plant. 2015:8:922-34.

34. Zhang G, Xu Q, Bian C, Tsai WC, Yeh CM, Liu K, Yoshida K, Zhang L, Chang S, Chen F, Shi Y, Su Y, Zhang Y, Chen L, Yin Y, Lin M, Huang H, Deng H, Wang Z, Zhu S, Zhao X, Deng C, Niu S, Huang J, Wang M, Liu G, Yang H, Xiao X, Hsiao YY, Wu W, Chen Y, Mitsuda N, Ohme-Takagi M, Luo Y, Van de Peer $Y$, Liu Z. The Dendrobium catenatum Lindl genome sequence provides insights into polysaccharide synthase, floral development and adaptive evolution. Sci Rep. 2016;6:19029.

35. Fan $H$, Wu Q, Wang X, Wu L, Cai Y, Lin Y. Molecular cloning and expression of 1-deoxy-D-xylulose-5-phosphate synthase and 1-deoxy-D-xylulose-5phosphate reductoisomerase in Dendrobium officinale. Plant Cell Tissue Organ Cult. 2016;125:381-5.

36. Zhu Y, Meng C, Zhu L, Li D, Jin Q, Song C, Cai Y, Fan H, Lin Y. Cloning and characterization of DoMYC2 from Dendrobium officinale. Plant Cell Tissue Organ Cult. 2017;129:533-41.

37. Wu Z, Jiang W, Chen S, Mantri N, Tao Z, Jiang C. Insights from the cold transcriptome and metabolome of Dendrobium officinale: global reprogramming of metabolic and gene regulation networks during cold acclimation. Front Plant Sci. 2016;7:1653.

38. Xing X, Cui SW, Nie S, Phillips GO, Goff HD, Wang Q. A review of isolation process, structural characteristics, and bioactivities of water-soluble polysaccharides from Dendrobium plants. Bioact Carbohydr Diet Fiber. 2013; 1:131-47.

39. Cheng CY, Krishnakumar V, Chan AP, Thibaud-Nissen F, Schobel S, Town CD. Araport11: a complete reannotation of the Arabidopsis thaliana reference genome. Plant J. 2017;89:789-804.

40. Acquadro A, Barchi L, Portis E, Mangino G, Valentino D, Mauromicale G, Lanteri S. Genome reconstruction in Cynara cardunculus taxa gains access to chromosome-scale DNA variation. Sci Rep. 2017;7:5617.

41. Xu H, Song J, Luo H, Zhang Y, Li Q, Zhu Y, Xu J, Li Y, Song C, Wang B, Sun W, Shen G, Zhang X, Qian J, Ji A, Xu Z, Luo X, He L, Li C, Sun C, Yan H, Cui G, Li X, Li X, Wei J, Liu J, Wang Y, Hayward A, Nelson D, Ning Z, Peters RJ, Qi $X$, Chen S. Analysis of the genome sequence of the medicinal plant Salvia miltiorrhiza. Mol Plant. 2016;9:949-52.

42. Keller TE, Yi SV. DNA methylation and evolution of duplicate genes. Proc Natl Acad Sci U S A. 2014;111:5932-7.

43. Pei L, Zhang L, Li J, Shen C, Qiu P, Tu L, Zhang X, Wang M. Tracing the origin and evolution history of methylation-related genes in plants. BMC Plant Biol. 2019;19:307.
44. Gu T, Ren S, Wang Y, Han Y, Li Y. Characterization of DNA methyltransferase and demethylase genes in Fragaria vesca. Mol Gen Genomics. 2016;291: 1333-45.

45. Ahmad F, Huang $X$, Lan $H$, Huma $T$, Bao $Y$, Huang J, Zhang $H$. Comprehensive gene expression analysis of the DNA (cytosine-5) methyltransferase family in rice (Oryza sativa L.). Genet Mol Res. 2014;13: 5159-72.

46. Qian Y, Xi Y, Cheng B, Zhu S. Genome-wide identification and expression profiling of DNA methyltransferase gene family in maize. Plant Cell Rep. 2014;33:1661-72.

47. Jeltsch A, Ehrenhofer-Murray A, Jurkowski TP, Lyko F, Reuter G, Ankri S, Nellen W, Schaefer M, Helm M. Mechanism and biological role of Dnmt2 in nucleic acid methylation. RNA Biol. 2017;14:1108-23.

48. Cao X, Jacobsen SE. Role of the Arabidopsis DRM methyltransferases in de novo DNA methylation and gene silencing. Curr Biol. 2002;12:1138-44.

49. Cheng J, Niu Q, Zhang B, Chen K, Yang R, Zhu JK, Zhang Y, Lang Z. Downregulation of RdDM during strawberry fruit ripening. Genome Biol. 2018;19:212

50. Gong Z, Morales-Ruiz T, Ariza RR, Roldán-Arjona T, David L, Zhu JK. ROS1, a repressor of transcriptional gene silencing in Arabidopsis, encodes a DNA glycosylase/lyase. Cell. 2002;111:803-14.

51. Zhang $X Q$, Fischer RL, Huh JH, Hsieh TF. The catalytic core of DEMETER guides active DNA demethylation in Arabidopsis. Proc Natl Acad Sci U S A. 2019;116:17563-71.

52. Ono A, Yamaguchi K, Fukada-Tanaka S, Terada R, Mitsui T, lida S. A null mutation of ROS1a for DNA demethylation in rice is not transmittable to progeny. Plant J. 2012;71:564-74.

53. La H, Ding B, Mishra GP, Zhou B, Yang H, Bellizzi Mdel R, Chen S, Meyers BC, Peng Z, Zhu JK, Wang G. A 5-methylcytosine DNA glycosylase/lyase demethylates the retrotransposon Tos 17 and promotes its transposition in rice. Proc Natl Acad Sci U S A. 2011;108:15498-503.

54. Yamaguchi-Shinozaki K, Shinozaki K. Organization of cis-acting regulatory elements in osmotic- and cold-stress-responsive promoters. Trends Plant Sci. 2005;10:88-94

55. Secco D, Wang C, Shou H, Schultz MD, Chiarenza S, Nussaume L, Ecker JR, Whelan J, Lister R. Stress induced gene expression drives transient DNA methylation changes at adjacent repetitive elements. eLife. 2015:4:e09343.

56. Cheng MC, Liao PM, Kuo WW, Lin TP. The Arabidopsis ethylene response factor 1 regulates abiotic stress-responsive gene expression by binding to different cis-acting elements in response to different stress signals. Plant Physiol. 2013;162:1566-82.

57. Murashige T, Skoog F. A revised medium for rapid growth and bioassays with tobacco tissue cultures. Physiol Plant. 1962;15:473-97.

58. Larkin MA, Blackshields G, Brown NP, Chenna R, McGettigan PA, McWilliam H, Valentin F, Wallace IM, Wilm A, Lopez R, Thompson JD, Gibson TJ, Higgins DG. Clustal W and Clustal X version 2.0. Bioinformatics. 2007;23: 2947-8.

59. Kumar S, Stecher G, Li M, Knyaz C, Tamura K. MEGA X: Molecular evolutionary genetics analysis across computing platforms. Mol Biol Evol. 2018:35:1547-9.

60. Saitou N, Nei M. The neighbor-joining method: a new method for reconstructing phylogenetic trees. Mol Biol Evol. 1987;4:406-25.

61. Kim D, Paggi JM, Park C, Bennett C, Salzberg SL. Graph-based genome alignment and genotyping with HISAT2 and HISAT-genotype. Nat Biotechnol. 2019;37:907-15.

62. Pertea M, Pertea GM, Antonescu CM, Chang TC, Mendell JT, Salzberg SL. StringTie enables improved reconstruction of a transcriptome from RNA-seq reads. Nat Biotechnol. 2015;33:290-5.

\section{Publisher's Note}

Springer Nature remains neutral with regard to jurisdictional claims in published maps and institutional affiliations. 